J. Math. Soc. Japan

Vol. 23, No. 1, 1971

\title{
Pseudogroups associated with the one dimensional foliation group $(\mathbf{I})$
}

\author{
By Richard M. KOCH
}

(Received May 29, 1970)

\section{INTRODUCTION}

Recent authors dealing with the classification of pseudogroups have treated the case where the group of first order terms is irreducible. Here the simplest of the reducible cases is treated; a classification is given of all pseudogroups whose first order terms generate the entire one dimensional foliation group $\left(\begin{array}{cc}a & b_{i} \\ 0 & d^{i}{ }_{j}\end{array}\right)$.

These pseudogroups are classified modulo equivalence by coordinate changes in $R^{N}$; there are then 19 major classes of such pseudogroups, of which 2 are families of pseudogroups parameterized by a real constant, and 3 are families parameterized by a positive integral constant.

The classification proceeds in two steps. Step $I$ is a classification of certain graded Lie algebras; such algebras appear in most pseudogroup theories. Step II is a solution of the equivalence problem and the actual derivation of the pseudogroups; here use is made of a theory due to R.C. Gunning at Princeton of pseudogroups defined by differential equations with constant coefficients.

Since Gunning has not published a detailed description of his theory, the first chapter of this paper contains a summary of his results. This theory is more restrictive than many recent treatments, but has the advantage of keeping formulas attractively explicit. Gunning considers the terms of order $\leqq n$ in the power series expansions of $C^{\infty}$ functions at some fixed point $x$; these form the elements of a group $\tilde{G}_{x}^{n}$. Those terms in $\tilde{G}_{x}^{n}$ coming from the expansion of the functions of a pseudogroup $\Gamma$ form a subgroup $G_{x}^{n}$ of $\tilde{G}_{x}^{n}$. If the differential equations defining $\Gamma$ have constant coefficients, this subgroup $G^{n}$ does not vary over the manifold, and so can be used to define $\Gamma$. Hence $\Gamma$ can be analyzed indirectly using Lie group methods on $G^{n}$.

For simplicity, Gunning omits terms of order 0 ; thus all pseudogroups are transitive. If local coordinates are chosen, the group $\tilde{G}^{n}$ can be given very explicitly. In particular, $\widetilde{G}^{1}$ is just $G L(N, R)$, and $G^{1}$ is precisely the 
group of all first order terms $\left(\frac{\partial f^{i}}{\partial x_{j}}\right)$. The classification problem can then be stated as follows: given the first order terms $G^{1}$, find all possible pseudogroups $\left\{G^{n}\right\}$ built over this first order group.

If a new coordinate system is chosen given by a coordinate transformation $\phi$, a new pseudogroup $\psi^{-1} \circ \Gamma \circ \psi$ is formed; this pseudogroup is naturally considered equivalent to $\Gamma$, and the above classification problem is stated modulo this equivalence relation.

We will constantly use the results of the classification when $G^{\mathbf{1}}=G L(N, R)$ (see Guillemin and Sternberg, [3]); these results are given at the end of chapter I. All of the results in this paper hold in two cases: one where $C^{\infty}$ real manifolds are involved, and all algebras and groups are real, the other where complex analytic manifolds are involved, and all algebras and groups are complex. For convenience I shall write only of the first case and let the reader fill in the details of the second.

The results of this paper were obtained in my thesis, written under Gunning's direction; I am grateful to him for help and encouragement.

\section{CHAPTER I: THE THEORETICAL FRAMEWORK}

\section{A. Locally-Flat Continuous Pseudogroups}

1. $\tilde{G}^{n}$

Suppose $f=\left(f^{1}, \cdots, f^{N}\right)$ is a $C^{\infty}$ map from $R^{N}$ to $R^{N}$. If $x \in R^{N}$, we can consider

$$
\left\langle\left.\frac{\partial f^{i}}{\partial x_{j}}\right|_{x},\left.\frac{\partial^{2} f^{i}}{\partial x_{j_{1}} \partial x_{j_{2}}}\right|_{x}, \cdots,\left.\frac{\partial^{n} f^{i}}{\partial x_{j_{1}} \cdots \partial x_{j_{n}}}\right|_{x}\right\rangle=\left\langle\xi_{j}^{i}, \cdots, \xi_{j_{1} \cdots j_{n}}^{i}\right\rangle .
$$

Notice that $\xi^{i}{ }_{j_{1} \cdots j_{k}}$ belongs to the space $T^{k}(N, R)$ of tensors fully symmetric in the lower indices. If $f$ is a local diffeomorphism, the matrix $\xi_{j}^{i}$ is nonsingular.

Let $\tilde{G}^{n}(N, R)$ be the set of all elements $\left\langle\xi^{i}, \cdots, \xi^{i}{ }_{j_{1} \cdots j_{n}}\right\rangle$ with $\xi^{i}{ }_{j}$ nonsingular, $\xi^{i}{ }_{j_{1} \cdots j_{k}} \in T^{k}(N, R)$. For each local diffeomorphism $f: R^{N} \rightarrow R^{N}$ and $x \in R^{N}$ there is an associated element $\eta_{x}(f) \in \tilde{G}^{n}$, described above. It is possible to make $\tilde{G}^{n}$ into a (Lie) group so that $\eta_{x}(f \circ g)=\eta_{g(x)}(f) \circ \eta_{x}(g)$. We wish to describe this group operation explicitly. Let $\vartheta=\xi \circ \nu$; clearly we expect $\vartheta^{i}{ }_{j_{1} \cdots j_{n}}$ to be a sum of terms of the form

$$
\sum_{k_{1} \cdots k_{q}} \xi_{k_{1} \cdots k_{q}}^{i} \nu^{k_{1}}{ }_{j_{1} \cdots j_{1}} \nu^{k_{2}}{ }_{j_{a_{1}+1} \cdots j_{a_{1}+a_{2}}} \cdots \nu^{k_{q_{j}}}{ }_{a_{a_{1}+\cdots+a_{q-1} \cdots j_{n}}} \text {. }
$$

However this expression is not necessarily symmetric in $j_{1}, \cdots, j_{n}$; we introduce an operator $\mathcal{S}$ which, roughly speaking, is a "minimal symmetrization" 
operator. For example,

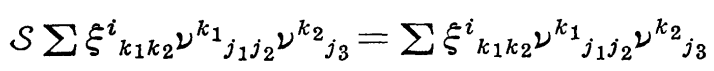

$$
\begin{aligned}
& +\sum \xi_{k_{1} k_{2}}^{i} \nu^{k_{1}}{ }_{j_{1} j_{3}} \nu^{k_{2}}{ }_{j_{2}}+\sum \xi_{k_{1} k_{2}}^{i} \nu_{j_{2} j_{3}}^{k_{1}} \nu_{j_{1}}^{k_{2}} \text {. }
\end{aligned}
$$

The reader can easily write down the general definition of $\mathcal{S}$.

THEOREM 1. If $\vartheta=\xi \circ \nu$,

In particular

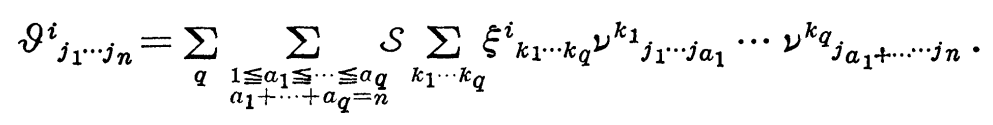

$$
\begin{aligned}
& \vartheta_{j}^{i}=\sum_{k} \xi_{k}^{i} \nu_{j}^{k} \\
& \vartheta_{j_{1} j_{2}}^{i}=\sum_{k} \xi^{i}{ }_{k} \nu^{k}{ }_{j_{1} j_{2}}+\sum_{k_{1} k_{2}} \xi_{{ }_{k_{1} k_{2}} \nu^{k_{1}}{ }_{j_{1}} \nu^{k_{2}}{ }_{j_{2}}} \\
& \vartheta_{j_{1} j_{2} j_{3}}^{i}=\sum_{k} \xi_{k}^{i} \nu^{k}{ }_{j_{1} j_{2} j_{3}}+\mathcal{S} \sum_{k_{1} k_{2}} \xi_{k_{1} k_{2}}^{i} \nu^{k_{1}}{ }_{j_{1}} \nu^{k_{2}}{ }_{j_{2} j_{3}} \\
& +\sum_{k_{1} k_{2} k_{3}} \xi_{k_{1} k_{2} k_{3}}^{i} \nu^{k_{1}} \nu_{j_{1}} \nu^{k_{2}}{ }_{j_{2}} \nu^{k_{3}}{ }_{j_{3}} .
\end{aligned}
$$

We shall in the main deal with Lie algebras rather than Lie groups. The Lie algebra $\tilde{g}^{n}$ of $\tilde{G}^{n}$ can be identified with all $\left\langle A_{\tilde{G}_{j}}^{i}, \cdots, A_{j_{1} \cdots j_{n}}^{i}\right\rangle, A_{j_{1} \cdots j_{k}}$ $\in T^{k}(N, R)$, in such a way that if $\xi^{i}{ }_{j_{1} \cdots j_{k}}(t)$ is a path in $\tilde{G}^{n}$ with $\xi(0)=$ identity, then $\xi^{*}\left(\frac{d}{d t}\right)=\left.\frac{d}{d t} \xi^{i}{ }_{j_{1} \cdots j_{k}}(t)\right|_{t=0}=A_{j_{1} \cdots j_{k}}^{i}$. Moreover, we have

THEOREM 2. If $C=[A, B]$,

$$
\begin{aligned}
& C^{i}{ }_{j_{1} \cdots j_{n}}=\sum_{k} \sum_{\lambda}\left\{A_{j_{1} \cdots k \cdots j_{n}}^{i} B_{j_{\lambda}}{ }^{i}-B_{j_{1} \cdots k \cdots j_{n}}^{i} A_{j_{\lambda}}^{k}\right\} \\
& +\sum_{k} \sum_{\lambda=1}^{n-1} \mathcal{S}\left\{A^{i}{ }_{k j_{1} \cdots j_{\lambda-1}-1} B^{k}{ }_{j_{\lambda} \cdots j_{n}}-B^{i}{ }_{k j_{1} \cdots j_{\lambda-1}} A^{k}{ }_{j_{\lambda \cdots j_{n}}}\right\}
\end{aligned}
$$

and in particular

$$
\begin{aligned}
& C^{i}{ }_{j}=\sum\left\{A^{i}{ }_{k} B^{k}{ }_{j}-B^{i}{ }_{k} A^{k}{ }_{j}\right\} \\
& C_{j_{1} j_{2}}^{i}=\sum\left\{A^{i}{ }_{k} B^{k}{ }_{j_{1} j_{2}}-B^{i}{ }_{k} A^{k}{ }_{j_{1} j_{2}}\right\}+\mathcal{S} \sum\left\{A_{j_{1} k}^{i} B^{k}{ }_{j_{2}}-B^{i_{j_{1} k}} A^{k}{ }_{j_{2}}\right\} \\
& C_{j_{1} j_{2} j_{3}}^{i}=\sum\left\{A_{k}^{i} B^{k}{ }_{j_{1} j_{2} j_{3}}-B^{i}{ }_{k} A^{k}{ }_{j_{1} j_{2} j_{3}}\right\} \\
& +\mathcal{S} \sum\left\{A_{j_{1} k}^{i} B_{j_{2} j_{3}}^{k}-B_{j_{1} k}^{i} A_{j_{2} j_{3}}^{k}\right\} \\
& +\mathcal{S} \sum\left\{A_{j_{1} j_{2} k}^{i} B_{j_{3}}^{k}-B_{j_{1} j_{2} k} A_{j_{3}}^{k}\right\} \text {. }
\end{aligned}
$$

Finally, we have a series of obvious maps $\sigma$ from $\tilde{G}^{n}$ to $\tilde{G}^{n-1}$,

$$
\sigma:\left\langle\xi_{j}^{i}, \cdots, \xi_{j_{1} \cdots j_{n}}^{i}\right\rangle \longrightarrow\left\langle\xi_{j}^{i}, \cdots, \xi_{j_{1} \cdots j_{n-1}}^{i}\right\rangle \text {. }
$$

Of course $\sigma\left(\eta_{x}^{n}(f)\right)=\eta_{x}^{n-1}(f)$. The $\sigma$ maps induce corresponding maps between Lie algebras, also denoted $\sigma$. 


\section{Definition and Fundamental Properties of Pseudogroups}

A differential equation with no terms of order 0 has the form

$$
\left\{F_{k}\left(-\frac{\partial \psi_{-}^{i}}{\partial x_{j}},-\frac{\partial^{2} \psi^{i}}{\partial x_{j_{1}} \partial x_{j_{2}}}, \cdots, \frac{\partial^{n} \psi^{i}}{\partial x_{j_{1}} \cdots \partial x_{j_{n}}}\right)=0\right\} \quad 1 \leqq k \leqq m
$$

we then define a subset $G^{n}{ }_{x}$ of $\tilde{G}^{n}$ by

$$
G^{n}{ }_{x}=\left\{\left\langle\xi_{j}^{i}, \cdots, \xi_{j_{1} \cdots j_{n}}^{i}\right\rangle\left|F_{k}\left(\xi_{j}^{i}, \cdots, \xi_{j_{1} \cdots j_{n}}^{i}\right)\right|_{x}=0\right\} \text {. }
$$

Clearly $\psi$ solves the differential equation at $x \Leftrightarrow \eta_{x}(\phi) \in G^{n}{ }_{x}$.

If the differential equation has constant coefficients, $G^{n}{ }_{x}$ does not depend on $x$ and we can write simply $G^{n}$. If $G^{n}$ is a group, the solutions of the differential equation form a pseudogroup since $\eta_{x}(\psi \circ \vartheta)=\eta_{\vartheta(x)}(\psi) \circ \eta_{x}(\vartheta)$. These remarks lead to the following definition:

Definition. Let $\Gamma$ be a $C^{\infty}$ pseudogroup on a manifold $M$. We call $\Gamma$ a continuous locally-flat pseudogroup if at each point of $M$ it is possible to choose a local coordinate neighborhood $\left(U, x_{1}, \cdots, x_{N}\right)$ and connected Lie subgroups $G^{n}$ of $\tilde{G}^{n}(N, R)$, so that

(1) $f \in \Gamma \mid \mathcal{U} \Leftrightarrow \eta_{x}(f) \in G^{n}$ for all $x \in \mathcal{U}$, and all $n$;

(2) every $\xi \in G^{n}$ can be realized by $\eta_{x}(f)$ for some $x \in U, f \in \Gamma \mid \mathcal{U}$;

(3) the natural maps $\sigma: G^{n} \rightarrow G^{n-1}$ are continuous.

The natural map in (3) is defined since (2) clearly implies that the maps $\sigma: \tilde{G}^{n} \rightarrow \tilde{G}^{n-1}$ take $G^{n}$ to $G^{n-1}$. The condition that $G^{n}$ be connected is chosen purely for convenience; a weaker assumption could certainly be made. The purpose of (3) is to insure that the $G^{n}$ 's are given a consistent topology.

Since we shall discuss only pseudogroups of the above type in the remainder of this paper, the term "pseudogroup" will henceforth be used for "locally-flat continuous pseudogroup". We will only deal with the local situation and so always think of $M$ as an open neighborhood of the origin in $R^{N}$.

It should be noted that (1) automatically guarantees that $\left\{f \mid \eta_{x}(f) \in G^{n}\right.$ for all $x \in \mathcal{U}$ and all $n\}$ is a pseudogroup; condition (2) puts very severe limitations on $G^{n}$.

Since by this definition $G^{n}$ determines the pseudogroup $\Gamma$, we now shift our attention to $G^{n}$; since $G^{n}$ is connected, it is sufficient to study the Lie algebra $g^{n}$ of $G^{n}$.

The above conditions limit the possibilities for $g^{n}$; we give below certain necessary conditions that $g^{n}$ yield a pseudogroup. In the particular cases we discuss, it will be a consequence of the classification theorem that these necessary conditions are also sufficient.

LEMMA 1. If $\left\{g^{n}\right\}$ gives a pseudogroup, the map $\sigma: g^{n} \rightarrow g^{n-1}$ is onto. 
Proof. This is an obvious consequence of the definition above.

Definition. Let $1 \leqq k \leqq N$. Define $\tau_{k}: T^{r}(N, R) \rightarrow T^{r-1}(N, R)$ by $\tau_{k}(\xi)^{i}{ }_{j_{1} \cdots j_{r-1}}$ $=\xi^{i}{ }_{k j_{1} \cdots j_{r-1}}$. Similarly define $\tau_{k}: \tilde{g}^{n} \rightarrow \tilde{g}^{n-1}$ by $\left\langle\tau_{k}(A)^{i}{ }_{j}, \cdots, \tau_{k}(A)^{i}{ }_{j_{1} \cdots j_{n-1}}\right\rangle=\left\langle A^{i}{ }_{k j}\right.$, $\left.\cdots, A^{i}{ }_{k j_{1} \cdots j_{n-1}}\right\rangle$.

DEFINITION. Let $g^{n-1}$ be a linear subspace of $\tilde{g}^{n-1}$. Then $\Lambda^{n} g^{n-1}$, a linear subspace of $\tilde{g}^{n}$, is the set of all $A$ in $\tilde{g}^{n}$, such that

(1) $\sigma(A) \in g^{n-1}$

(2) $\tau_{k}(A) \in g^{n-1}$ for all $1 \leqq k \leqq N$.

LEMMA 2. Let $G^{n}$ define a pseudogroup, and suppose $\xi \in G^{n}$. Then $\tau_{k}(\xi)$ - $\sigma\left(\xi^{-1}\right) \in g^{n-1}$. (Composition is in the sense of the group operation in $G^{n-1}$.)

Proof. Since $\xi \in G^{n}$, we can find $f \in \Gamma$ such that $\eta_{0}(f)=\xi, \eta_{x}(f) \in G^{n}$ for all $x \in \mathcal{U}$. Thus $\phi(t)=\left[\eta_{(0 \cdots t \cdots 0)}(f)\right] \circ\left[\eta_{0}(f)\right]^{-1} \in G^{n}$ for $|t|<\varepsilon$. But $\psi(0)$ is the identity, so $\left.\frac{d}{d t} \psi(t)\right|_{t=0} \in g^{n}$, so $\sigma\left(\left.\frac{d}{d t} \psi(t)\right|_{t=0}\right) \in g^{n-1}$.

But

$$
\eta_{(0 \cdots t \cdots 0)}(f)=\left(\frac{\partial f^{i}}{\partial x_{j}}(0 \cdots t \cdots 0), \frac{\partial^{2} f^{i}}{\partial x_{j_{1}} \partial x_{j_{2}}}(0 \cdots t \cdots 0), \cdots\right)
$$

and so

$$
\left.\frac{d}{d t} \eta_{(0 \cdots t \cdots 0)}(f)\right|_{t=0}=\left(\frac{\partial^{2} f^{i}}{\partial x_{k} \partial x_{j}}, \frac{\partial^{3} f^{i}}{\partial x_{k} \partial x_{j_{1}} \partial x_{j_{2}}}, \cdots\right)
$$

the rest is obvious by inspection.

LEMMA 3. Let $\left\{g^{n}\right\}$ define a pseudogroup and $A \in g^{n}$. Then $\tau_{k}(A) \in g^{n-1}$.

PROoF. If $A \in g^{n}$, we can find $\xi(t)$ in $G^{n}$, $\frac{d \xi}{d t}=A$. Then $\phi(t)=\tau_{k}(\xi(t))$ . $\sigma[\xi(t)]^{-1} \in g^{n-1}$ for all $t$. Hence since $g^{n-1}$ is a linear subspace, $\frac{d}{d t}-\left.\phi(t)\right|_{t=0}$ $\in g^{n-1}$. This is

$$
\left\{\left.\frac{d}{d t} \tau_{k}(\xi(t))\right|_{t=0}\right\} \circ \sigma(\xi(0))^{-1}+\left.\left\{\tau_{k}(\xi(0))\right\} \circ \frac{d}{d t} \sigma(\xi(t))^{-1}\right|_{t=0} .
$$

Since $\sigma(\xi(0))^{-1}=$ the identity and $\tau_{k}(\xi(0))=0$ the lemma is clear.

LEMMA 4. If $\left\{g^{n}\right\}$ defines a pseudogroup, $g^{n} \cong \Lambda^{n} g^{n-1}$.

ProOF. A consequence of the above lemmas.

THEOREM 3. If $g^{1}, g^{2}, \cdots, g^{n}, \ldots$ yields a pseudogroup,

(1) $\sigma: g^{n} \rightarrow g^{n-1}$ is onto

(2) $g^{n} \cong \Lambda^{n} g^{n-1}$.

Let $G^{1}$ be a connected Lie subgroup of $G L(N, R)$; we want to find all pseudogroups with $G^{1}$ as the group of first order terms. This suggests:

Classification Problem. Given $g^{1}$ a Lie subalgebra of $g^{1}(N, R)$, find all series of subalgebras $g^{n} \cong \tilde{g}^{n}$ satisfying the above conditions. 


\section{The Kernel Sequence}

Since $g^{n} \rightarrow g^{n-1}$ is onto, we have an exact sequence

$$
0 \longrightarrow k^{n} \longrightarrow g^{n} \longrightarrow g^{n-1} \longrightarrow 0
$$

where $k^{n} \leqq T^{n}(N, R) . \quad k^{n}$ is called the $n^{\text {th }}$ kernel of the pseudogroup; the sequence of $k^{n}$ s is called the kernel sequence. By definition, $k^{1}=g^{1}$.

In solving the above classification problem, we propose first to classify all possible kernel sequences and then to classify all extensions $0 \rightarrow k^{n} \rightarrow$ ? $\rightarrow g^{n-1} \rightarrow 0$.

LEMMA 5. $\Lambda^{k} k^{n-1} \supseteqq k^{n}$.

PROOF. If $\left\langle 0, \cdots, 0, A^{i}{ }_{j_{1} \cdots j_{n}}\right\rangle \in k^{n} \cong g^{n}$, then $\tau_{k}\left\langle 0, \cdots, 0, A_{{ }_{j_{1} \cdots j_{n}}}^{{ }}\right\rangle=\langle 0, \cdots$, $\left.A^{i}{ }_{k j_{1} \cdots j_{n-1}}\right\rangle \in g^{n-1}$, so it belongs to $k^{n-1}$, hence the result.

Consider $A=\left\langle 0, \cdots, 0, A_{j_{1} \cdots j_{k}}^{i}, 0, \cdots, 0\right\rangle \in g^{n}, B=\left\langle 0, \cdots, 0, B_{j_{1} \cdots j_{l}}, 0, \cdots, 0\right\rangle$ $\in g^{n}$.

LEMMA 6. $[A, B]_{j_{1} \cdots j_{r}}$ is non-zero only if $r=k+l-1$.

Proof. Examine the explicit form of $[A, B]$ given previously and notice that $[A, B]_{j_{1} \cdots j_{n}}^{i}$ involves only terms with $m$ indices multiplied by terms with $n-m+1$ indices.

LEMMA 7. $\left[k^{n}, k^{m}\right] \cong k^{n+m-1}$.

PROOF. A corollary of lemma 6.

THEOREM 4. The kernel sequence $g^{1}=k^{1}, k^{2}, \cdots, k^{n}, \cdots$ must satisfy

(1) $\left[k^{n}, k^{m}\right] \subseteq k^{n+m-1}$

(2) $k^{n} \subseteq \Lambda^{n} k^{n-1}$.

Conversely, if this is true and we let $g^{n}$ be the trivial extension of $g^{n-1}$ via $k^{n}$, the $g^{n}$ satisfy the requirements of theorem 3.

Proof. If $g^{n}$ has the form described in the last sentence, $g^{n}=\left\langle k^{1}, k^{2}, \cdots\right.$, $\left.k^{n}\right\rangle$. It suffices if this is a subalgebra of $g^{n}$, a result holding because of Lemma 7. Hence

Classification Problem. Find all sequences $k^{n} \cong T^{n}(N, R)$, such that

(1) $k^{1}=g^{1}$

(2) $\left[k^{l}, k^{m}\right] \leqq k^{l+m-1}$

(3) $k^{n} \subseteq \Lambda^{n} k^{n-1}$.

LEMmA 8. If $A \in k^{1}$, and $B \in k^{n}$, then

$$
[A, B]_{j_{1} \cdots j_{n}}^{i}=\sum A^{i}{ }_{k} B^{k}{ }_{j_{1} \cdots j_{n}}-\sum B_{j_{1} \cdots k \cdots j_{n}}^{i} A_{j_{\lambda}}^{k} .
$$

Proof. A calculation, from the results obtained previously.

We denote the above by $[d \rho(A) B]_{j_{1} \cdots j_{n}}^{i}$. It is clearly the Lie algebra representation corresponding to the representation of $G L(N, R)$ on $T^{n}(N, R)$ given by 


$$
[\rho(\xi) \varphi]_{j_{1} \cdots j_{n}}^{i}=\sum \xi^{i}{ }_{k} \varphi^{k}{ }_{s_{1} \cdots s_{n}}\left(\xi^{-1}\right)^{s_{1}}{ }_{j_{1}} \cdots\left(\xi^{-1}\right)^{s_{n} n_{j_{n}}} .
$$

Hence :

LEMMA 9. $k^{n}$ must be an invariant subspace of $T^{n}(N, R)$ under the standard representation of $G^{1}$.

(For more on this representation, see chapter 4 of Weyl [9] or chapter 5 of Boerner [2].)

Interestingly enough, the kernel sequences appear in almost all approaches to pseudogroup theory. See, for example, Guillemin and Sternberg [3], Kobayashi and Nagano [7].

\section{Further Results on $g^{n}$ and $k^{n}$}

Lemma 10. If $A \in \tilde{g}^{n+1}, B \in \tilde{g}^{n+1}$, then $\tau_{k}[A, B]=\left[\tau_{k}(A), \sigma(B)\right]+[\sigma(A)$, $\left.\tau_{k}(B)\right]+\sum_{r}\left\{B^{r}{ }_{k} \tau_{r}(A)-A^{r}{ }_{k} \tau_{r}(B)\right\}$.

PROOF. Calculate from previous formulas.

LEMMA 11. Let $k^{1}, k^{2}, \cdots, k^{n}$ satisfy

(1) $\left[k^{r}, k^{s}\right] \leqq k^{r+s-1}$ whenever $r \leqq n, s \leqq n, r+s-1 \leqq n$

(2) $k^{r} \leqq \Lambda^{r} k^{r-1}$ whenever $r \leqq n$.

Then $k^{1}, k^{2}, \cdots, k^{n}, \Lambda^{n+1} k^{n}, \Lambda^{n+2} \Lambda^{n+1} k^{n}, \cdots$ satisfies all requirements for a sequence of kernels.

PROOF. Define $k^{r}=\Lambda^{r} \Lambda^{r-1} \cdots \Lambda^{n+1} k^{n}$ for $r>n$. Since $k^{s} \cong \Lambda^{s} k^{s-1}$ for all $s$, we need only prove that if $A \in k^{m}, B \in k^{r}$, then $[A, B] \in k^{m+r-1}$. We prove this by double induction, the principal induction being on $m$. If $m=1$ and $r \leqq n$, then $m+r-1 \leqq n$ and the result is true by assumption. Retaining the assumption $m=1$, we apply a sub-induction to $r$. If $r>n$ we need only prove $\tau_{k}[A, B] \in k^{r-1}$ by definition of $k^{r}$. But we can apply lemma 10 since $k^{n} \leqq \tilde{g}^{n+a}$ for $a \geqq 0$; thus $\tau_{k}[A, B]=\left[\tau_{k}(A), B\right]+\left[A, \tau_{k}(B)\right]+\sum_{r}\left\{B^{r}{ }_{k} \tau_{r} A-A^{r}{ }_{k} \tau_{r} B\right\}$. But $A \in k^{1}$, so $\tau_{k}(A)=0 ; B \in k^{r}$ so by definition $\tau_{k}(B) \in k^{r-1}$; then by subinduction $\left[A, \tau_{k}(B)\right] \in k^{r-1}$; finally $\sum A^{s}{ }_{k} \tau_{s}(B)$ is a linear combination of elements of $k^{r-1}$, so in $k^{r-1}$. This completes the sub-induction step.

Assume the lemma for $m \leqq k$, and let $m=k+1(m>1)$. We again use a sub-induction on $r$. If $r=1$, we are done by the above, reversing the roles of $A$ and $B$. If $r>1$, and $m+r-1 \leqq n$, then $m \leqq n, r \leqq n$, so $[A, B] \in k^{m+r-1}$ by assumption. If $m+r-1>n$, we must prove $\tau_{s}[A, B]$ in $k^{m+r-2}$ or $\left[\tau_{s} A, B\right]$ $+\left[A, \tau_{s} B\right]+\sum_{w}\left\{B^{w}{ }_{s} \tau_{w} A-A^{w}{ }_{s} \tau_{w} B\right\}$ in $k^{m+r-2}$. Since $r>1, m>1, B^{i}{ }_{j}=A^{i}{ }_{j}=0$, and this last term vanishes; $\left[\tau_{s} A, B\right] \in k^{m+r-2}$ by induction, $\left[A, \tau_{s} B\right] \in k^{m+r-2}$ by sub-induction.

The above lemma provides a way of constructing a kernel sequence when part of one is at hand. It is a theorem of Kuranishi (the prolongation theo- 
rem) that every kernel sequence eventually becomes $\Lambda^{n+1} k^{n}, \Lambda^{n+2} \Lambda^{n+1} k^{n}$, etc. We do not need that theorem here (indeed our results will constitute a verification of the theorem in a particular case). However, we shall now show how in the presence of such a kernel sequence the group $G^{n}$ determines the pseudogroup (so $G^{n+1}$, etc., are irrelevant).

LEMMA 12. If $g^{n}$ is an algebra, so is $\Lambda^{n+1} g^{n}$.

Proof. Since $[A, B]_{j_{1} \cdots j_{k}}$ depends only on terms of degree $\leqq k$, if $A$ and $B$ are in $\Lambda^{n+1} g^{n}$ then $\sigma[A, B] \in g^{n}$. It suffices then if $\tau_{k}[A, B] \in g^{n}$ for fixed $k$. But this is clear from lemma 10 .

THEOREM 5. Let $k^{1}, \cdots, k^{n}, \Lambda^{n+1} k^{n}, \cdots$ be a kernel sequence, and let $g^{1}, \cdots$, $g^{n}$ be a sequence of subalgebras which fit this kernel sequence and satisfy $g^{k} \cong \Lambda^{k} g^{k-1}$. Then if $g^{1}, \cdots, g^{n}$ can be extended at all to give a complete series of algebras, the extension must be $\Lambda^{n+1} g^{n}, \Lambda^{n+2} \Lambda^{n+1} g^{n}, \cdots$. However, for this to work we must have

$$
\begin{gathered}
\sigma: \Lambda^{n+1} g^{n} \longrightarrow g^{n} \\
\sigma: \Lambda^{n+2} \Lambda^{n+1} g^{n} \longrightarrow \Lambda^{n+1} g^{n} \\
\vdots
\end{gathered}
$$

all onto. (That this is not necessarily so will become apparent later; this condition will lead to integrability conditions for certain differential equations.)

PROOF. $\quad 0 \rightarrow k^{n} \rightarrow g^{n} \rightarrow g^{n-1} \rightarrow 0$ is exact. Then consider

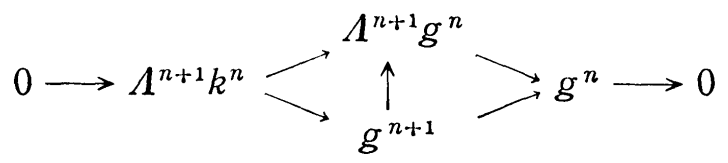

(The vertical map is given by inclusion.) Since $g^{n+1} \rightarrow g^{n}$ is required to be onto, $\Lambda^{n+1} g^{n} \rightarrow g^{n}$ must be onto. Conversely, if this holds and if the top sequence is exact, the 5-lemma guarantees that $g^{n+1}=\Lambda^{n+1} g^{n}$. But the exactness of the top sequence is clear; indeed if $\left\langle 0, \cdots, 0, A^{i}{ }_{j_{1} \cdots j_{n+1}}\right\rangle \in \Lambda^{n+1} g^{n}$, for each fixed $k\left\langle 0, \cdots, 0, A^{i}{ }_{k j_{1} \cdots j_{n}}\right\rangle$ is in $g^{n}$, so $A^{i}{ }_{{ }_{j j_{1} \cdots j_{n}}} \in k^{n}$, so $A_{{ }_{1} \cdots j_{n+1}}^{i} \in \Lambda^{n+1} k^{n}$.

COROLLARY. Let $k^{1}, \cdots, k^{n}, \Lambda^{n+1} k^{n}, \cdots$ be a kernel sequence corresponding to two pseudogroups $G^{1}, \cdots, G^{k}, \cdots$ and $\check{G}^{1}, \cdots, \check{G}^{k}, \cdots$ and suppose $G^{1}=\check{G}^{1}, \cdots$, $G^{n}=\check{G}^{n}$. Then the two pseudogroups are identical.

Proof. $g^{1}=\check{g}^{1}, \cdots, g^{n}=\check{g}^{n}$, and the theorem then forces $g^{n+s}=\check{g}^{n+s}$, and so $G^{n+s}=\check{G}^{n+s}$.

In this sense, for kernels of the above type finitely many $g^{k}$ 's determine the pseudogroup. 


\section{A Well-Known Special Case}

Suppose $G^{1} \cong O(n)$, the orthogonal group. Then $A^{i}{ }_{j} \in g^{1} \Rightarrow A^{i}{ }_{j}=-A^{j}{ }_{i}$. Hence $\left\langle A^{i}{ }_{j}, A^{i}{ }_{j_{1} j_{2}}\right\rangle \in \Lambda^{2} g^{1} \Rightarrow A^{i}{ }_{k j}=-A^{j}{ }_{k i}=-A^{j}{ }_{i k}=A^{k}{ }_{i j}=A^{k}{ }_{j i}=-A^{i}{ }_{j k}=-A^{i}{ }_{k j}$, so $A^{i}{ }_{k j}=0$. As $g^{2} \subseteq \Lambda^{2} g^{1}$, we conclude that $g^{2}=\left\langle A^{i}{ }_{j}, 0\right\rangle$, and in general that $g^{n}=\left\langle A^{i}{ }_{j}, 0, \cdots, 0\right\rangle$; and so $G^{n}=\left\langle G^{1}, 0, \cdots, 0\right\rangle$. Thus the only possible pseudogroup is

$$
\left\{y_{i}=\sum_{j} a_{i j} x_{j}+b_{i}\right\} \quad a_{i j} \in G^{1} .
$$

This example, included only as a curiosity, illustrates how the conditions on $g^{n}$ can severely limit the possible pseudogroups.

\section{The Equivalence Relation}

Let $M$ and $N$ be manifolds, $\psi: M \rightarrow N$ a diffeomorphism, and $\Gamma$ a pseudogroup on $M$. Then $\psi \circ \Gamma \circ \psi^{-1}$ (the meaning of this notation is obvious) is clearly a pseudogroup on $N$.

In particular, if $M$ and $N$ are open neighborhoods of 0 on $R^{N}$, and $\phi(0)$ $=0$, we have a method of going from one pseudogroup $\Gamma$ to another $\psi \circ \Gamma \circ \psi^{-1}$, both defined in some neighborhood of the origin. Then:

Definition. Let $\Gamma$ and $\Gamma^{\prime}$ be (locally-flat continuous) pseudogroups defined (in some neighborhood of the origin) by $G^{n}$ and $\check{G}^{n}$ respectively. We say these pseudogroups are equivalent if a diffeomorphism $\psi: \mathcal{U} \rightarrow \mathcal{C}$ can be found of sufficiently small neighborhoods of the origin, $\phi(0)=0$, so that $\Gamma\left|\mathcal{Q} \cap \bar{Q}=\left(\psi \circ \Gamma^{1} \circ \psi^{-1}\right)\right| \mathcal{Q} \cap \mathcal{V}$.

We intend to show in chapter 3 that for the pseudogroups we classify, the various extensions

$$
0 \longrightarrow k^{n} \longrightarrow \text { ? } \longrightarrow g^{n-1} \longrightarrow 0
$$

all yield equivalent pseudogroups, so we may take the trivial extension as an example of the pseudogroup produced by $k^{n}$.

\section{Conclusion}

The above remarks constitute in outline Gunning's general theory. Further developments, below, hold only if special assumptions on $g^{1}$ are made.

Clearly a number of questions remain unanswered; the most important is whether the conditions listed in Theorem 3 are sufficient. Also unanswered is the relation of the equivalence problem for pseudogroups to the extension problem for our algebras. In our case the question of extension of $g^{n}$ to $g^{n+1}$ via $k^{n+1}$ is only a matter of equivalent pseudogroups. 


\section{B. Further Developments}

1. The Case $E \in g^{1}$

Special formulas of a more precise nature hold in case the identity matrix $E$ is in the Lie algebra $g^{1}$.

Suppose we first examine $0 \rightarrow k^{2} \rightarrow g^{2} \rightarrow g^{1} \rightarrow 0$. Then there must be an element $\langle E, \varphi\rangle \in g^{2}$, since the last map is onto. Since $g^{2} \subseteq \Lambda^{2} g^{1}, \varphi \in \Lambda^{2} k^{1}$. But we can add to $\varphi$ any element of $k^{2}$, and so pick only one element in an equivalence class $\tilde{\varphi} \in \frac{\Lambda^{2} k^{1}}{k^{2}}$. Now this $\varphi$ determines $g^{2}$ completely.

LEMMA 13.

$$
g_{\varphi}^{2}=\left\{\left\langle A^{i}{ }_{j}, A_{j_{1} j_{2}}^{i}\right\rangle \mid A_{j_{1} j_{2}}^{i}=-\left[d \rho\left(A^{i}{ }_{j}\right) \varphi\right]_{j_{1} j_{2}}^{i}+k^{2}\right\} .
$$

Proof. A simple calculation.

It should be emphasized that $g^{2}{ }_{\varphi}$ need not be an element of a chain, since $\Lambda^{3} g^{2}{ }_{\varphi} \rightarrow g^{2}{ }_{\varphi}$ need not be onto. If $\varphi=0$, we have the trivial extension, which always is part of a chain.

Definition. $\theta_{\varphi}{ }^{2}: \tilde{G}^{2} \rightarrow T^{2}(N, R)$ is defined by

$$
\left[\theta^{2}{ }_{\varphi}\left(\xi_{j}^{i}, \xi_{j_{1} j_{2}}^{i}\right)\right]_{j_{1} j_{2}}^{i}=\sum \xi_{k_{1} k_{2}}^{i}\left(\xi^{-1}\right)^{k_{1}}{ }_{j_{1}}\left(\xi^{-1}\right)^{k_{2}}{ }_{j_{2}}+\left[\rho\left(\xi_{j}^{i}\right) \varphi\right]_{j_{1} j_{2}}^{i}-\varphi_{j_{1} j_{2}}^{i}
$$

LEMMA 14.

$$
\theta_{\varphi}^{2}(\xi \circ \eta)=\theta^{2}(\xi)+\rho(\xi) \theta_{\varphi}^{2}(\eta) .
$$

Proof. A calculation.

THEOREM 6.

$$
G_{\varphi}^{2}=\left\{\left(\xi_{j}^{i}, \xi^{i}{ }_{j_{1} j_{2}}\right) \mid \xi^{i}{ }_{j} \in G^{1}, \theta^{2}{ }_{\varphi}\left(\xi^{i}{ }_{j}, \xi^{i}{ }_{j_{1} j_{2}}\right) \in k^{2}\right\}
$$

is the Lie group generated by $g^{2}{ }_{\varphi}$; if $G^{1}$ is connected, so is $G^{2}$.

PROOF. This follows immediately from the lemma above and a little thought. Connectivity is immediate, for

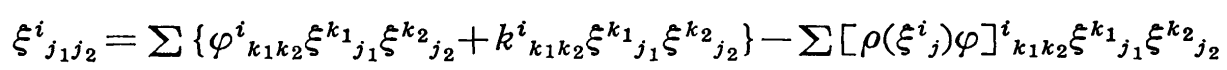

so a path $\psi(t)$ joining $\xi^{i}{ }_{j}$ to the identity in $G^{1}$ and a path $k(t)$ joining $k^{i}{ }_{k_{1} k_{2}}$ to 0 in $k^{2}$ yield a path $\xi_{j_{1} j_{2}}^{i}(t)$ joining $\left\langle\xi^{i}{ }_{j}(1), \xi_{j_{1} j_{2}}^{i}(1)\right\rangle$ to $\langle E, 0\rangle$.

THEOREM 7. The pseudogroup defined by $G^{2}{ }_{\varphi}$ is equivalent to the pseudogroup defined by $G^{2}$, provided there is a local diffeomorphism $\psi: \mathcal{U} \rightarrow \mathcal{Q}, \mathcal{U}$ and $\checkmark$ open neighborhoods of 0 in $R^{N}, \phi(0)=0$, such that

(1) $\frac{\partial \psi^{i}}{\partial x_{j}} \in G^{1}$

(2) $\theta_{0}^{2}\left(\eta_{x}(\psi)\right)-\varphi \in k^{2}$ for each $x \in U$.

PROOF. We must find $\psi$ so that $\frac{\partial}{\partial x_{i}}\left(\phi g \psi^{-1}\right)^{j} \in G^{1}$, and $\theta^{2}{ }_{\varphi}\left(\eta_{x}\left(\psi g \psi^{-1}\right)\right) \in k^{2}$ 
for all $x \Leftrightarrow \frac{\partial g^{j}}{\partial x_{i}} \in G^{1}$ and $\theta_{0}^{2}\left(\eta_{x}(g)\right) \in k^{2}$, for all $x$.

But if $\frac{\partial \phi^{i}}{\partial x_{j}} \in G^{1}$, the first part holds. Then the required result is $\theta^{2}{ }_{\varphi}\left(\eta_{x}\left(\psi g \psi^{-1}\right)\right)=\theta_{0}^{2}\left(\eta_{x}\left(\psi g \psi^{-1}\right)\right)+\rho\left(\eta_{x}\left(\psi g \psi^{-1}\right)\right) \varphi-\varphi \in k^{2} \Leftrightarrow \theta^{2}{ }_{0}\left(\eta_{x} g\right) \in k^{2}$.

The left hand side is

$$
\theta_{0}^{2}\left(\eta_{g \psi^{-1}(x)}(\psi) \circ \eta_{\psi^{-1}(x)}(g) \circ \eta_{x}\left(\psi^{-1}\right)\right)+\rho\left(\eta_{x}\left(\psi g \psi^{-1}\right)\right) \varphi-\varphi .
$$

If we apply Lemma 14, this becomes

$$
\begin{aligned}
& \theta_{0}^{2}\left(\eta_{g \psi^{-1}(x)}(\psi)\right)+\rho\left(\eta_{g \psi^{-1}(x)}(\psi)\right) \theta^{2}{ }_{0}\left(\eta_{\psi^{-1}(x)}(g)\right) \\
& \quad+\rho\left(\eta_{g \psi^{-1}(x)}(\psi)\right) \rho\left(\eta_{\psi^{-1}(x)}(g)\right) \theta^{2}{ }_{0}\left(\eta_{x}\left(\psi^{-1}\right)\right)+\rho\left(\eta_{x}\left(\psi g \psi^{-1}\right)\right) \varphi-\varphi .
\end{aligned}
$$

However,

$$
\begin{aligned}
& \theta^{2}{ }_{0}\left(\eta_{\psi^{-1}(x)}\left(\psi^{-1} \circ \psi\right)\right)=\theta^{2}{ }_{0}(\langle E, 0\rangle) \\
& =0=\theta_{0}{ }_{0}\left(\eta_{x}\left(\psi^{-1}\right)\right)+\rho\left(\eta_{x}\left(\psi^{-1}\right)\right) \theta_{0}^{2}\left(\eta_{\psi^{-1}(x)}(\psi)\right)
\end{aligned}
$$

so we may rewrite the left hand side as

$$
\begin{aligned}
\left\{\theta^{2}{ }_{0}\left(\eta_{g \psi^{-1}(x)}(\psi)\right)-\varphi\right\} & +\rho\left(\eta_{x}\left(\psi g \psi^{-1}\right)\right)\left\{\theta^{2}{ }_{0}\left(\eta_{\psi^{-1}(x)}(\psi)\right)-\varphi\right\} \\
& +\rho\left(\eta_{g \psi^{-1}(x)}(\psi)\right) \theta^{2}{ }_{0}\left(\eta_{\psi^{-1}(x)}(g)\right) .
\end{aligned}
$$

Now $k^{2}$ is invariant under the representation $d \rho$, so also under the representation $\rho$; since $\eta_{x}\left(\psi g \psi^{-1}\right) \in G^{1}$, the lemma is obvious.

LEMMA 15. Let $g^{n-1}=\left\{\left\langle A_{1}, \cdots, A_{n-1}\right\rangle \mid A_{1} \in k^{1}, \cdots, A_{n-1} \in k^{n-1}\right\}$. Then there exists $\varphi \in \Lambda^{n} k^{n-1} / k^{n}$ such that

$$
\begin{array}{r}
g^{n}=\left\{\left\langle A_{1}, \cdots, A_{n}\right\rangle \mid\left\langle A_{1}, \cdots, A_{n-1}\right\rangle \in g^{n-1}, A_{n}\right. \\
\left.=-\frac{1}{n-1}\left[d \rho\left(A_{1}\right) \varphi\right]+k^{n}\right\} .
\end{array}
$$

Proof. $\langle E, \cdots, 0\rangle \in g^{n-1}$, so $D==\langle E, 0, \cdots, 0, \varphi\rangle \in g^{n}$. Since $g^{n} \cong \Lambda^{n} g^{n-1}$, $\varphi \in \Lambda^{n} k^{n-1}$. But $\varphi-\tilde{\varphi} \in k^{n}$ implies $\langle E, 0, \cdots, 0, \tilde{\varphi}\rangle \in g^{n}$.

Now suppose $A=\left\langle A_{1}, 0, \cdots, 0, A_{n}\right\rangle \in g^{n}$. Then $[A, D]=\left\langle 0, \cdots, 0,\left[A_{n}, E\right]\right.$ $\left.+\left[A_{1}, \varphi\right]\right\rangle$. Hence $\left[A_{n}, E\right]=(n-1) A_{n}=-\left[A_{1}, \varphi\right]+k^{n}$, so the lemma holds in this case.

By induction, we assume the lemma if $A_{2}=\cdots=A_{k}=0$, and prove it if $A_{2}=\cdots=A_{k-1}=0$.

Indeed, then

$$
\begin{aligned}
{[A, D] } & =\left\langle 0_{1}, \cdots, 0_{k-1},\left[A_{k}, E\right], \cdots,\left[A_{n}, E\right]+\left[A_{1}, \varphi\right]\right\rangle \\
& =\left\langle 0_{1}, \cdots, 0_{k-1},(k-1) A_{k}, \cdots,(n-1) A_{n}+\left[A_{1}, \varphi\right]\right\rangle .
\end{aligned}
$$

Hence 


$$
(k-1) A-[A, D]=\left\langle(k-1) A_{1}, 0_{2}, \cdots, 0_{k}, \cdots,(k-1) A_{n}-(n-1) A_{n}-\left[A_{1}, \varphi\right]\right\rangle .
$$

We can apply the induction hypothesis to this element, and conclude that

$$
(k-n) A_{n}-\left[A_{1}, \varphi\right]=-\frac{1}{n-1}\left[(k-1) A_{1}, \varphi\right]+k^{n}
$$

or

$$
(k-n) A_{n}=\frac{n-1}{n-1}\left[A_{1}, \varphi\right]-\frac{k-1}{n-1}\left[A_{1}, \varphi\right]+k^{n},
$$

which implies the lemma in this case also.

Definition. $\theta_{\varphi}^{3}: \tilde{G}^{3} \rightarrow T^{3}(N, R)$ is defined by

LEMMA 16.

$$
\begin{aligned}
& \theta^{3}{ }_{\varphi}(\xi)=2 \sum \xi^{i}{ }_{k_{1} k_{2} k_{3}}\left(\xi^{-1}\right)^{k_{1}}{ }_{j_{1}}\left(\xi^{-1}\right)^{k_{2}}{ }_{j_{2}}\left(\xi^{-1}\right)^{k_{3}}{ }_{j_{3}} \\
& -\mathcal{S} \Sigma \theta^{2}{ }_{0}(\xi)^{i}{ }_{j_{1} k} \theta^{2}{ }_{0}(\xi)^{k}{ }_{j_{2} j_{3}}+\rho(\xi) \varphi-\varphi \text {. }
\end{aligned}
$$

$$
\theta_{\varphi}^{3}(\xi \circ \eta)=\theta_{\varphi}^{3}(\xi)+\rho(\xi) \theta_{\varphi}^{3}(\eta)+\left[\theta^{2}{ }_{0}(\xi), \rho(\xi) \theta_{0}^{2}(\eta)\right] .
$$

ProOF. A calculation.

THEOREM 8.

$$
G^{3}{ }_{\varphi}=\left\{\xi \mid \xi_{j}^{i} \in G^{1}, \theta^{2}{ }_{0}(\xi) \in k^{2}, \theta^{3}{ }_{\varphi}(\xi) \in k^{3}\right\} .
$$

PROOF. This is a group, for the composition of two elements is in it by the lemma and the fact that $k^{3}$ is invariant under $\rho$ and $\left[k^{2}, k^{2}\right] \subseteq k^{8}$. It is a connected Lie group for essentially the same reason that $G^{2}$ is a connected Lie group. Finally, it has the correct Lie algebra; indeed if

$$
\frac{d}{d t}-\xi(t)=A, \frac{d}{d t} \theta_{\varphi}^{3}(\xi(t))=2 A_{3}-\left[A_{1}, \varphi\right],
$$

so

$$
A_{3}=-\frac{1}{2}\left[A_{1}, \varphi\right]+k^{3} .
$$

THEOREM 9. The pseudogroup defined by $G^{3}{ }_{\varphi}$ is equivalent to the pseudogroup defined by $G^{3}$ provided there is a local diffeomorphism $\phi: \mathcal{U} \rightarrow \mathcal{V}, \mathcal{U}$ and $Q \mathcal{V}$ open neighborhoods of 0 in $R^{N}, \phi(0)=0$, such that

(1) $\phi$ is in the pseudogroup defined by $G^{2}{ }_{0}$

(2) $\theta_{0}^{3}\left(\eta_{x}(\phi)\right)-\varphi \in k^{3}$, for each $x \in \mathcal{U}$.

Proof. We want $\theta_{\varphi}^{3}\left(\eta_{x}\left(\psi f \psi^{-1}\right)\right) \in k^{3} \Leftrightarrow \theta_{0}^{3}\left(\eta_{x}(f)\right) \in k^{3}$. But

$$
\begin{aligned}
\theta_{\varphi}{ }_{\varphi}\left(\eta_{x}\left(\psi f \psi^{-1}\right)\right)= & \theta_{0}^{3}\left(\eta_{x}\left(\psi f \psi^{-1}\right)\right)+\rho\left(\eta_{x}\left(\phi f \psi^{-1}\right)\right) \varphi-\varphi \\
= & \theta_{0}^{3}\left(\eta_{f \psi^{-1}(x)}(\psi)\right)+\rho\left(\eta_{f \psi^{-1}(x)}(\psi)\right) \theta_{0}{ }_{0}\left(\eta_{x}\left(f \psi^{-1}\right)\right) \\
& +\left[\theta^{2}{ }_{0}\left(\eta_{f \psi^{-1}(x)}(\psi)\right), \rho\left(\eta_{f \psi^{-1}(x)}(\psi)\right) \theta_{0}{ }_{0}\left(\eta_{x}\left(f \psi^{-1}\right)\right)\right] \\
& +\rho\left(\eta_{x}\left(\psi f \psi^{-1}\right)\right) \varphi-\varphi
\end{aligned}
$$


But

$$
\begin{aligned}
& =\left\{\theta_{0}^{3}\left(\eta_{f \psi^{-1}(x)}(\psi)\right)-\varphi\right\}+\rho\left(\eta_{f \psi^{-1}(x)}(\psi)\right) \theta_{0}{ }_{0}\left(\eta_{\psi^{-1}(x)}(f)\right) \\
& +\left\{\rho\left(\eta_{f \psi^{-1}(x)}(\psi)\right) \rho\left(\eta_{\psi^{-1}(x)}(f)\right) \theta^{3}{ }_{0}\left(\psi^{-1}\right)+\rho\left(\eta_{x}\left(\psi f \psi^{-1}\right)\right) \varphi\right\} \\
& +\rho\left(\eta_{f \psi^{-1}(x)}(\psi)\right)\left[\theta^{2}{ }_{0}\left(\eta_{\psi^{-1}(x)}(f)\right), \rho\left(\eta_{\psi^{-1}}{ }_{(x)}(f)\right) \theta^{2}{ }_{0}\left(\eta_{x}\left(\psi^{-1}\right)\right)\right] \\
& +\left[\theta_{0}^{2}\left(\eta_{f \psi^{-1}(x)}(\psi)\right), \rho\left(\eta_{f \psi^{-1}}{ }_{(x)}(\psi)\right) \theta_{0}^{2}\left(\eta_{x}\left(f \psi^{-1}\right)\right)\right] .
\end{aligned}
$$

$$
\begin{aligned}
0=\theta_{0}^{3}\left(\eta_{x}\left(\psi \circ \psi^{-1}\right)\right)= & \theta_{0}^{3}\left(\eta_{\psi^{-1}(x)}(\psi)\right)+\rho\left(\eta_{\psi^{-1}}(x)(\psi)\right) \theta^{3}{ }_{0}\left(\psi^{-1}\right) \\
& \left.+\left[\theta_{0}{ }_{0}\left(\eta_{\psi^{-1}}(x)\right)\right), \rho\left(\eta_{\psi^{-1}(x)}(\psi)\right) \theta^{2}{ }_{0}\left(\eta_{x}\left(\psi^{-1}\right)\right)\right] .
\end{aligned}
$$

Also

$$
\theta_{0}^{2}\left(\eta_{x}\left(\psi^{-1}\right)\right)=-\rho\left(\eta_{x}\left(\psi^{-1}\right)\right) \theta_{0}^{2}\left(\eta_{\psi^{-1}}{ }_{(x)}(\psi)\right)
$$

so this last part vanishes and

$$
\theta_{0}^{3}\left(\eta_{x}\left(\psi^{-1}\right)\right)=-\rho\left(\eta_{x}\left(\psi^{-1}\right)\right) \theta^{3}{ }_{0}\left(\eta_{\psi^{-1}(x)}(\psi)\right),
$$

so the formula is

$$
\begin{aligned}
\theta_{\varphi}^{3}\left(\eta_{x}\left(\phi f \psi^{-1}\right)\right)= & \left\{\theta_{0}^{3}\left(\eta_{f \psi^{-1}(x)}(\psi)\right)-\varphi\right\} \\
& +\rho\left(\eta_{f \psi^{-1}(x)}(\psi)\right) \theta^{3}{ }_{0}\left(\eta_{\psi^{-1}(x)}(f)\right) \\
& -\rho\left(\eta_{x}\left(\phi f \psi^{-1}\right)\right)\left\{\theta^{3}{ }_{0}\left(\eta_{\psi^{-1}(x)}(\psi)\right)-\varphi\right\} \\
& +\rho\left(\eta_{f \psi^{-1}(x)}(\psi)\right)\left[\theta^{2}{ }_{0}\left(\eta_{\psi^{-1}(x)}(f)\right), \rho\left(\eta_{\psi^{-1}(x)}(f)\right) \theta^{2}{ }_{0}\left(\eta_{x}\left(\psi^{-1}\right)\right)\right] \\
& +\left[\theta^{2}{ }_{0}\left(\eta_{f \psi^{-1}(x)}(\psi)\right), \rho\left(\eta_{f \psi^{-1}(x)}(\psi)\right) \theta_{0}{ }_{0}\left(\eta_{x}\left(f \psi^{-1}\right)\right)\right]
\end{aligned}
$$

and since everything in sight is in $G^{2}{ }_{0}$ and $\left[k^{2}, k^{2}\right] \subseteq k^{3}$, the theorem is clear.

\section{The Classification of Pseudogroups for $G L(N, R)$}

By a previous remark, $k^{n}$ must be an invariant subspace of $T^{n}(N, R)$ under the standard representation of $G L(N, R)$. But this standard representation splits into two invariant subrepresentations:

DEFINITION.

$$
\begin{aligned}
& (1-\Omega)\left(T^{n}\right)=\left\{\xi_{j_{1} \cdots j_{n}}^{i} \in T^{n} \mid \sum \xi^{i}{ }_{k j_{1} \cdots j_{n-1}}=0\right\} \\
& \Omega\left(T^{n}\right)=\left\{\xi_{j_{1} \cdots j_{n}}^{i} \mid \xi^{i}{ }_{j_{1} \cdots j_{n}}=\sum \delta^{i}{ }_{j_{\lambda}} \eta_{j_{1} \cdots \hat{j}_{\lambda} \cdots j_{n}}\right. \text { for some } \\
& \\
& \eta_{j_{1} \cdots j_{n-1}} \in S^{n-1}(N, R), \text { the space of fully } \\
& \text { symmetric tensors }\} .
\end{aligned}
$$

LEMMA 17. These subspaces are irreducible invariant subspaces and

$$
T^{n}=(1-\Omega)\left(T^{n}\right) \oplus \Omega\left(T^{n}\right) \quad(N \geqq 2)
$$

PROoF. A standard computation. 
Hence $k^{n}$ can only be $T^{n},(1-\Omega)\left(T^{n}\right), \Omega\left(T^{n}\right)$, or 0 . The conditions $\left[k^{r}, k^{8}\right]$ $\subseteq k^{r+8-1}$ give still more restrictions, and one easily computes:

THEOREM 10. The following is a complete classification of all kernel sequences for $G L(N, R)$ :

$$
\begin{aligned}
& N=1: \quad g^{1} \quad k^{2} \quad k^{3} \quad k^{4} \quad k^{n} \\
& \text { C } \longrightarrow 0 \longrightarrow 0 \longrightarrow 0 \longrightarrow 0 \\
& C \longrightarrow 0 \longrightarrow 0 \longrightarrow 0 \\
& \longrightarrow C \longrightarrow C \longrightarrow C \\
& N \geqq 2: \quad 0 \longrightarrow 0 \longrightarrow 0 \longrightarrow 0
\end{aligned}
$$

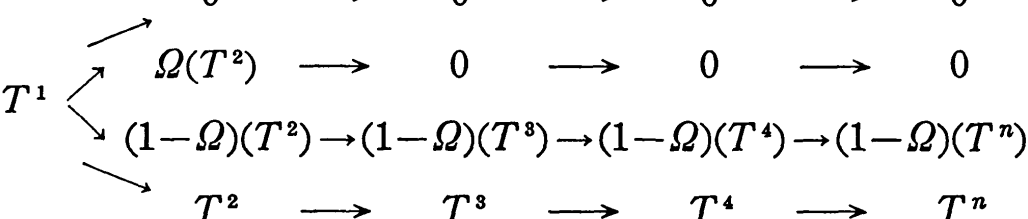

Next the equivalence problem must be solved. Here Gunning proves:

THEOREM 11. All extensions $0 \rightarrow k^{n} \rightarrow g^{n} \rightarrow g^{n-1} \rightarrow 0$ are equivalent to the trivial extension.

It is then a simple matter to compute the pseudogroups; we have:

THEOREM 12. The following is a complete list of all pseudogroups for $G L(N, R)$, up to equivalence:

$$
\begin{array}{ll}
N=1: \quad\{y=a x+b \\
& \left\{y=\frac{a x+b}{c x+d}\right. \\
& \{y=f(x) \\
& \left\{y_{i}=\sum a_{i j} x_{j}+b_{i}\right. \\
& \left\{y_{i}=\frac{\sum a_{i j} x_{j}+b_{i}}{\sum c_{j} x_{j}+d}\right. \\
& \left\{y_{i}=f_{i}\left(x_{1}, \cdots, x_{n}\right) \quad \operatorname{det}\left(\frac{\partial f^{i}}{\partial x_{j}}\right)=\right.\text { constant } \\
& \left\{y_{i}=f_{i}\left(x_{1}, \cdots, x_{n}\right)\right.
\end{array}
$$

\section{CHAPTER II : THE KERNEL SEQUENCES}

\section{Preliminaries to the Classification Theorem}

As a preliminary to the calculation which follows, I collect here several useful formulas. The Lie algebra of the foliation group consists of all matrices of the form $\left(\begin{array}{cc}a & b_{i} \\ 0 & d^{i}{ }_{j}\end{array}\right) \quad(2 \leqq i \leqq N ; 2 \leqq j \leqq N)$. The "largest" kernel 
sequence is just $k^{1}, \Lambda^{2} k^{1}, \cdots$. An element of $\Lambda^{n} \Lambda^{n-1} \cdots \Lambda^{2} k^{1}$ is a tensor $A^{i}{ }_{j_{1} \cdots j_{n}}$; we write this tensor in the form

$$
\left(\begin{array}{cccc}
A^{1}{ }_{11 \cdots 1} & A^{1}{ }_{1 \cdots 1 j_{1}} & A^{1}{ }_{1 \cdots 1 j_{1} j_{2}} & A^{1}{ }_{j_{1} \cdots j_{n}} \\
A^{i}{ }_{11 \cdots 1} & A^{i \cdots 1 j_{1}} & A^{i}{ }_{1 \cdots 1 j_{1} j_{2}} & A_{{ }_{1} \cdots j_{n}}^{i}{ }^{i}{ }^{1}
\end{array}\right)
$$

where $i, j_{1}, \cdots, j_{n} \geqq 2$.

LEMMA 18. $\Lambda^{n} \Lambda^{n-1} \cdots \Lambda^{2} k^{1}=$ the set of all

$$
\left(\begin{array}{cccc}
e & e_{j_{1}} & e_{j_{1} j_{2}} & e_{j_{1} \cdots j_{n}} \\
0 & 0 & 0 & e^{i}{ }_{j_{1} \cdots j_{n}}
\end{array}\right)
$$

where each element is symmetric in all lower indices. That is, $A_{{ }_{j_{1} \cdots j_{n}}}^{{ }_{1}}=0$ if $i \geqq 2$ and at least one $j_{1}=1$. (We are letting $A^{1}{ }_{1 \cdots 1 j_{1} \cdots j_{k}}=e_{j_{1} \cdots j_{k}}\left(j_{i} \geqq 2\right), A^{i}{ }_{j_{1} \cdots j_{n}}$ $=e^{i}{ }_{j_{1} \cdots j_{n}}\left(i \geqq 2, j_{i} \geqq 2\right)$.)

PROOF. Induction on $n$. If $n=1$, the lemma is true by the definition of $k^{1}$. If the lemma holds for $n-1$, we have

$$
\begin{aligned}
& A^{i}{ }_{1 j_{1} \cdots j_{n-1}}=\left(\begin{array}{cccc}
{ }^{(1)} e & { }^{(1)} e_{j_{1}} & { }^{(1)} e_{j_{1} j_{2}} & { }^{(1)} e_{j_{1} \cdots j_{n-1}} \\
0 & 0 & 0 & { }^{(1)} e_{j_{1} \cdots j_{n-1}}
\end{array}\right) \\
& A^{i}{ }_{k j_{1} \cdots j_{n-1}}=\left(\begin{array}{cccc}
{ }^{(k)} e & { }^{(k)} e_{j_{1}} & { }^{(k)} e_{j_{1} j_{2}} & { }^{(k)} e_{j_{1} \cdots j_{n-1}} \\
0 & 0 & 0 & { }^{(k)} e^{i}{ }_{j_{1} \cdots j_{n-1}}
\end{array}\right)
\end{aligned}
$$

But $A^{1}{ }_{11 \cdots 1 j_{1} \cdots j_{n-r}}={ }^{(1)} e_{j_{1} \cdots j_{n-r}}={ }^{(j)} e_{j_{2} \cdots j_{n-r}}$; therefore the top row of the bottom box, shifted one to the right, is the same as the top row of the top box. Since ${ }^{(1)} A_{j_{1} \cdots j_{n}}=0$, the lemma also holds for $n$.

LEMMA 19.

$$
\begin{aligned}
& d \rho\left(\begin{array}{ll}
a & a_{i} \\
0 & b^{i}{ }_{j}
\end{array}\right)\left[\left(\begin{array}{ccc}
e & e_{i} & e_{i j} \\
0 & 0 & d^{i}{ }_{j k}
\end{array}\right)\right] \\
& =\left(\begin{array}{ccc}
-a e & \left\{-a_{j} e-\sum e_{k} b^{k}{ }_{j}\right\}\left\{a e_{j_{1} j_{2}}-a_{j_{1}} e_{j_{2}}-a_{j_{2}} e_{j_{1}}-\sum e_{j_{1} k} b^{k}{ }_{j_{2}}-\sum e_{j_{2} k} b^{k}{ }_{j_{1}}+\sum a_{k} d^{i}{ }_{j_{1} j_{2}}\right\} \\
0 & 0 & (d \rho(b)[d])^{i}{ }_{j_{1} j_{2}}
\end{array}\right)
\end{aligned}
$$

\begin{tabular}{|c|c|c|c|c|c|}
\hline & $k^{2}$ & & $k^{3}$ & & $k^{n}$ \\
\hline (1) & 0 & $\longrightarrow$ & 0 & $\longrightarrow$ & 0 \\
\hline
\end{tabular}

Proof. Calculate.

Of course $(d \rho(b)[d])^{i}{ }_{j_{1} j_{2}}$ means that $b^{i}{ }_{j}$ and $d^{i}{ }_{j_{1} j_{2}}$ are to be treated as tensors in $R^{N-1}$.

The purpose of this chapter is to prove the following theorem:

THEOREM 13. The following is a complete list of all kernels for $\left(\begin{array}{ll}a & a_{i} \\ 0 & b^{i}{ }_{j}\end{array}\right)$. 


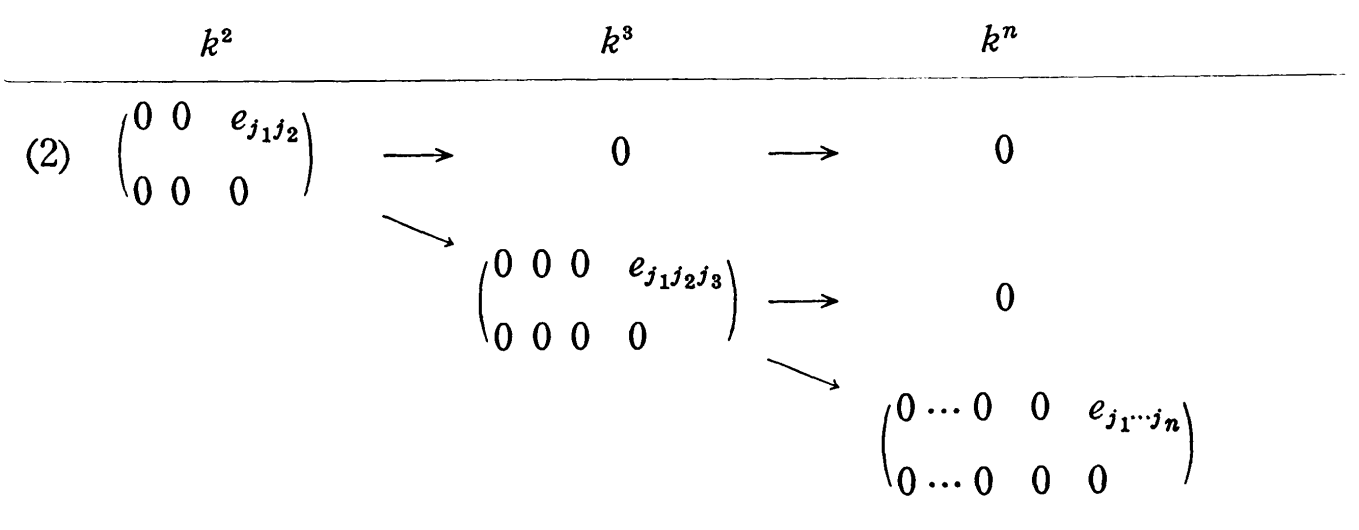

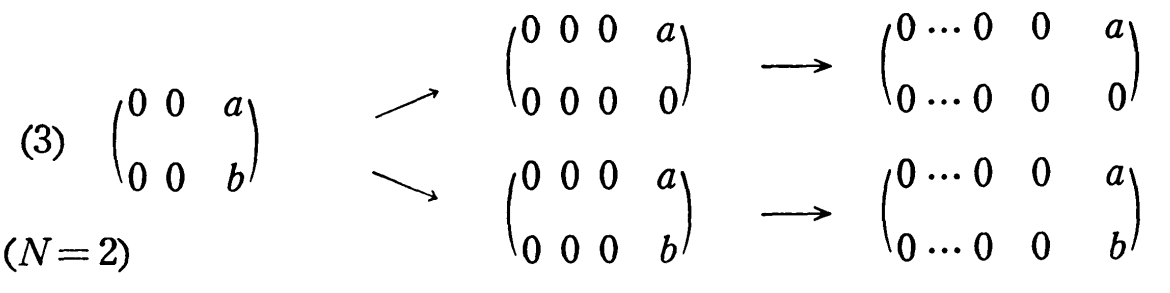

(4) $\left(\begin{array}{llr}0 & 0 & e_{j_{1} j_{2}} \\ 0 & 0 & \Omega\left(T^{2}\right)\end{array}\right) \rightarrow\left(\begin{array}{llll}0 & 0 & 0 & e_{j_{1} j_{2} j_{3}} \\ 0 & 0 & 0 & 0\end{array}\right) \rightarrow\left(\begin{array}{lllll}0 & \ldots & 0 & 0 & e_{j_{1} \cdots j_{n}} \\ 0 & \ldots & 0 & 0 & 0\end{array}\right)$ $(N>2)$

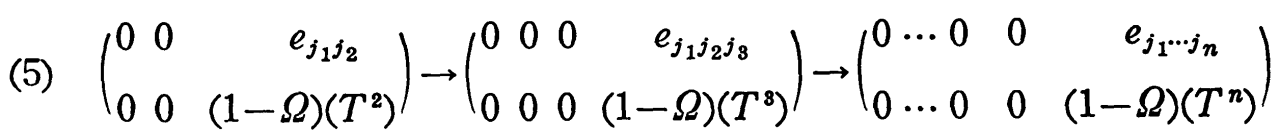
$(N>2)$

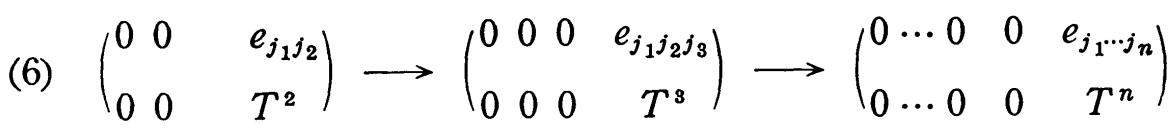
$(N>2)$

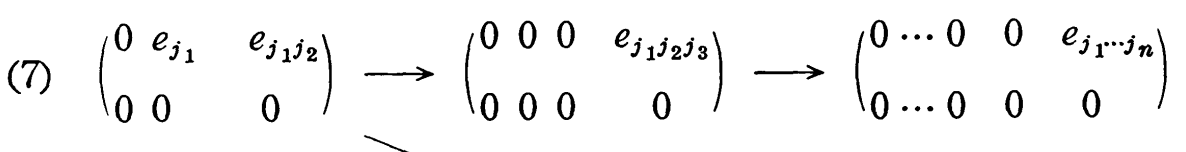

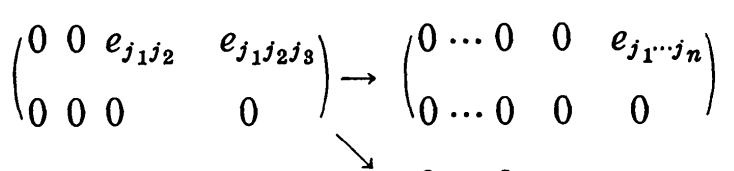

$$
\begin{aligned}
& \left(\begin{array}{lll}
0 \cdots 0 & e_{j_{1} \cdots j_{n-1}} & e_{j_{1} \cdots j_{n}} \\
0 \cdots 0 & 0 & 0
\end{array}\right)
\end{aligned}
$$




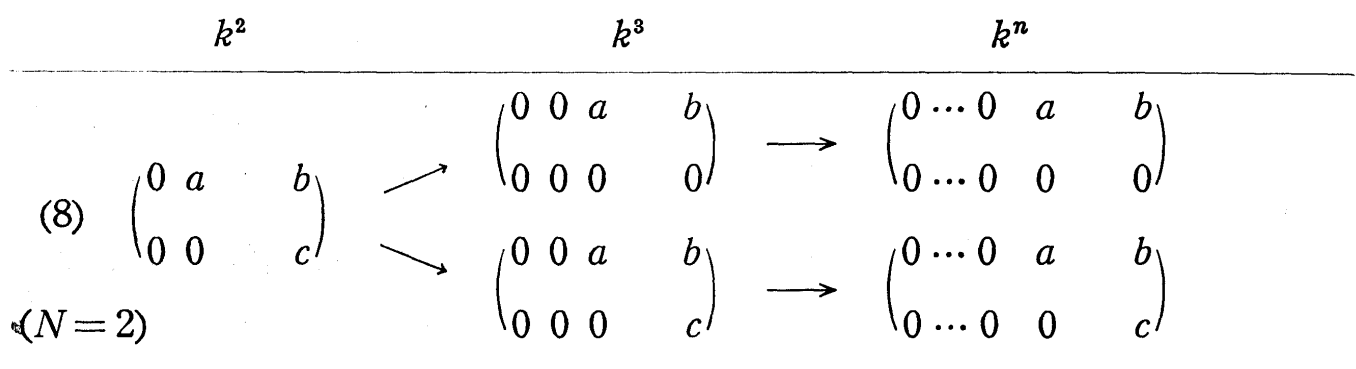

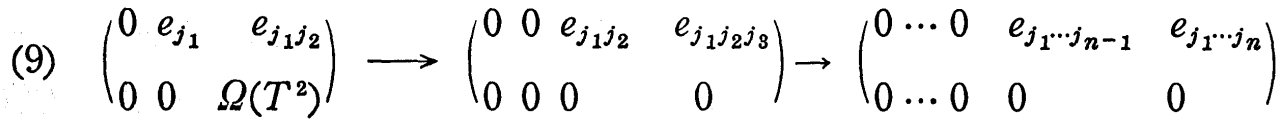
$(N>2)$

(10) $\left(\begin{array}{ccc}0 & e_{j_{1}} & e_{j_{1} j_{2}} \\ 0 & 0 & (1-\Omega)\left(T^{2}\right)\end{array}\right) \rightarrow\left(\begin{array}{cccc}0 & 0 & e_{j_{1} j_{2}} & e_{j_{1} j_{2} j_{3}} \\ 0 & 0 & 0 & (1-\Omega)\left(T^{3}\right)\end{array}\right) \rightarrow\left(\begin{array}{ccccc}0 & \cdots & 0 & e_{j_{1} \cdots j_{n-1}} & e_{j_{1} \cdots j_{n}} \\ 0 & \cdots & 0 & 0 & (1-\Omega)\left(T^{n}\right)\end{array}\right)$ $(N>2)$

(11) $\left(\begin{array}{ccc}0 & e_{j_{1}} & e_{j_{1} j_{2}} \\ 0 & 0 & T^{2}\end{array}\right) \rightarrow\left(\begin{array}{cccc}0 & 0 & e_{j_{1} j_{2}} & e_{j_{1} j_{2} j_{3}} \\ 0 & 0 & 0 & T^{3}\end{array}\right) \rightarrow\left(\begin{array}{lllll}0 & \cdots & 0 & e_{j_{1} \cdots j_{n-1}} & e_{j_{1} \cdots j_{n}} \\ 0 & \cdots & 0 & 0 & T^{n}\end{array}\right)$ $(N>2)$

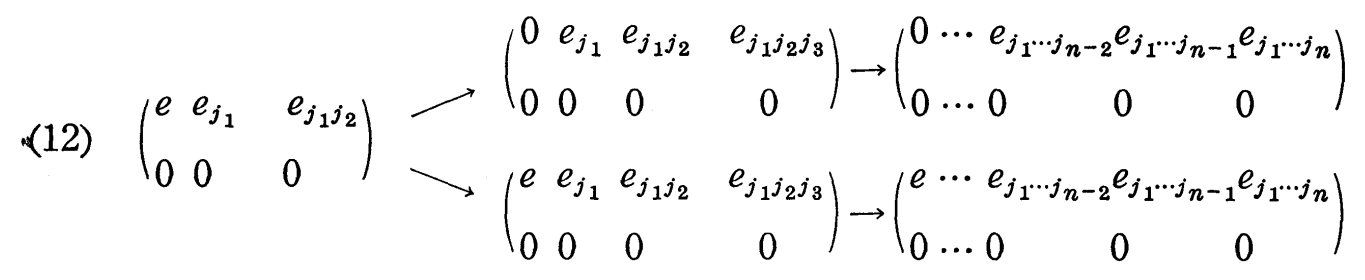

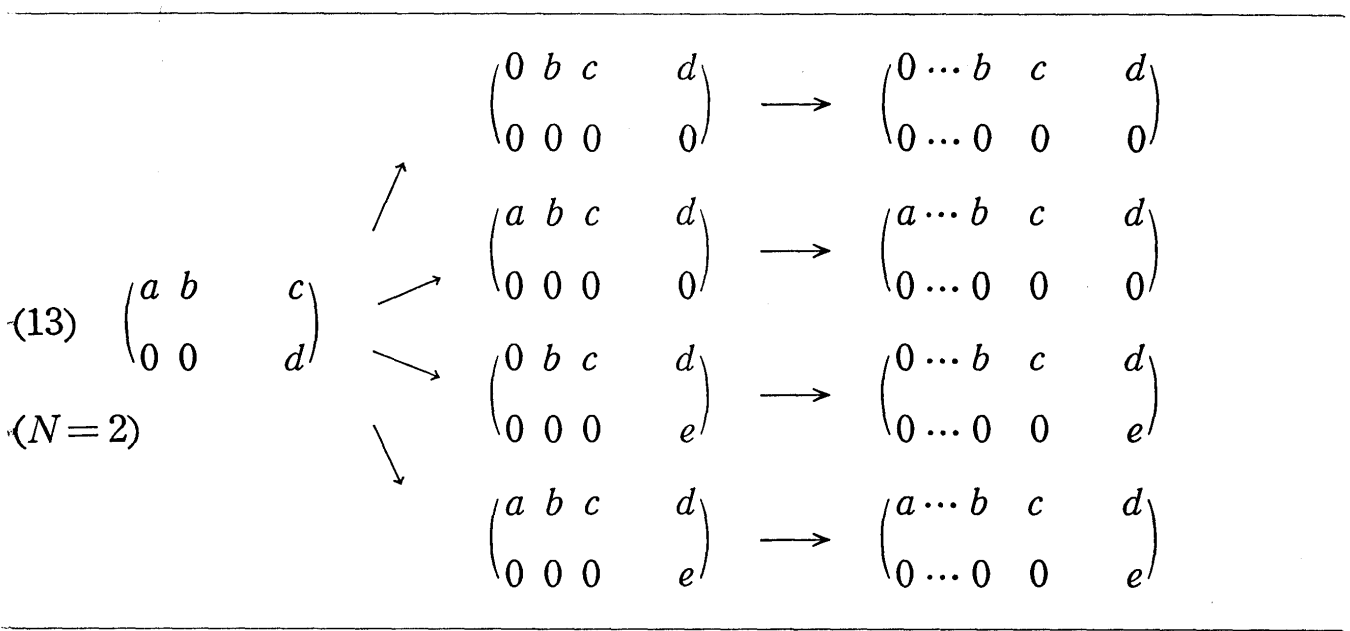




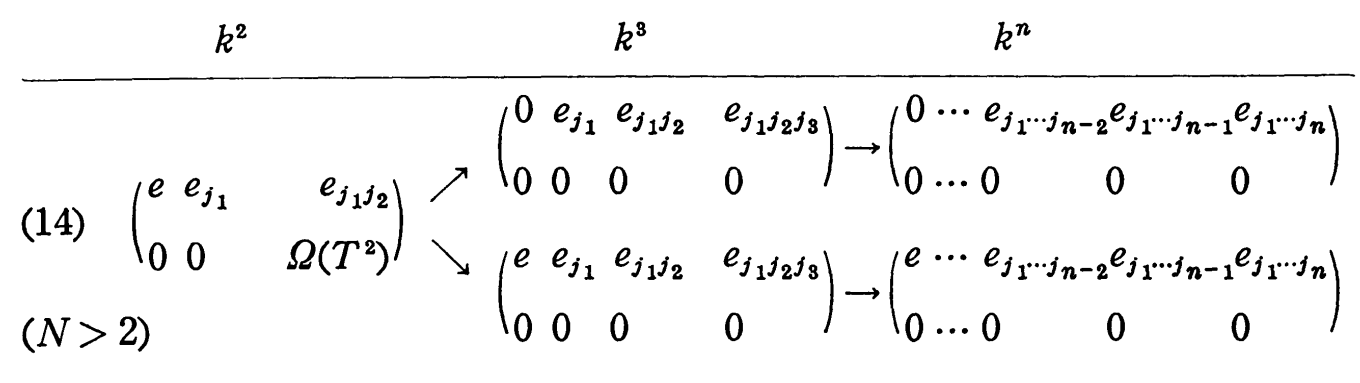

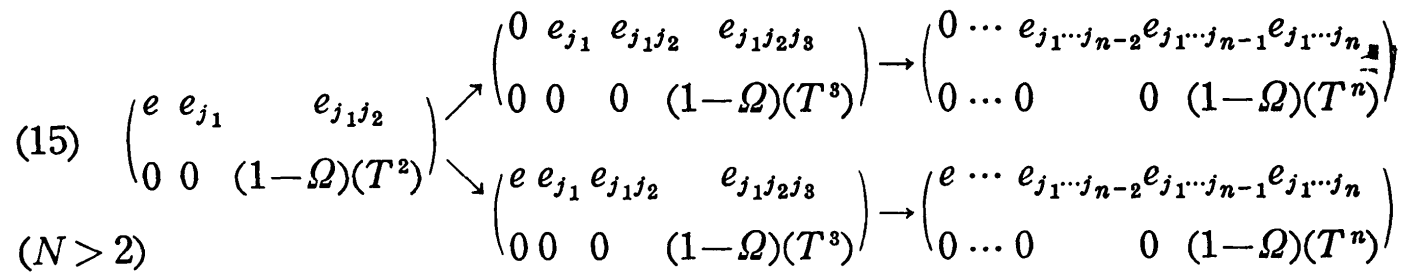

$(16)\left(\begin{array}{lll}e & e_{j_{1}} & e_{j_{1} j_{2}} \\ 0 & 0 & T^{2}\end{array}\right) \searrow\left(\begin{array}{llll}0 & e_{j_{1}} & e_{j_{1} j_{2}} & e_{j_{1} j_{2} j_{3}} \\ 0 & 0 & 0 & T^{3}\end{array}\right)$
$(N>2)$

$$
\left(\begin{array}{lll}
0 \lambda d^{(2) j}{ }_{j j} & e_{j_{1} j_{2}} \\
0 & 0 & T^{2}
\end{array}\right) \longrightarrow \quad \Lambda^{3} k^{2} \quad \longrightarrow \quad \Lambda^{n} \cdots \Lambda^{8} k^{2}
$$

$(N>2)$

$$
\begin{aligned}
& \left(d^{(2)}=\text { component of } d^{i}{ }_{j_{1} j_{2}} \text { in } \Omega\left(T^{2}\right), d_{j_{1} j_{2}}^{i} \in T^{2}\right) \\
& (\lambda \neq 0)
\end{aligned}
$$

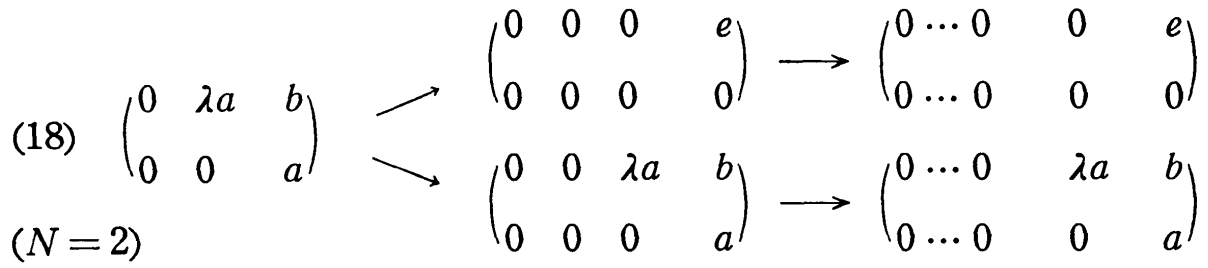

( $\lambda$ fixed, $\lambda \neq 0$ )

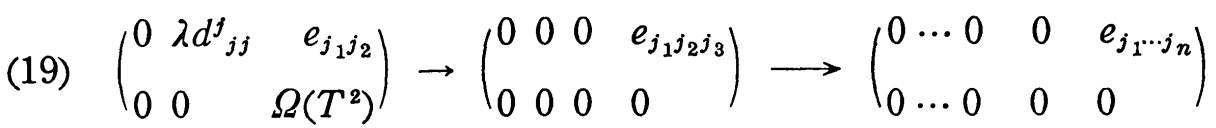
$(N>2)$ ( $\lambda$ fixed, $\lambda \neq 0$ ) 
However: In (18) and (19) we have special cases if $\lambda=\frac{n-1}{2} \quad(n=2,3$, $4, \cdots)$. If $n=2$, this takes the form of a new $k^{2}$ sequence:

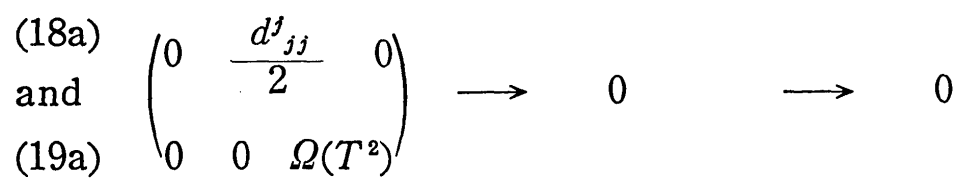

If $n \geqq 3$, we can replace the $n^{\text {th }}$ kernel by zero and so get:

\begin{tabular}{|c|c|c|}
\hline & $k^{l}(l<n)$ & $k^{l}(l>n)$ \\
\hline $\begin{array}{l}(18 \mathrm{~b}) \\
(N=2)\end{array}$ & 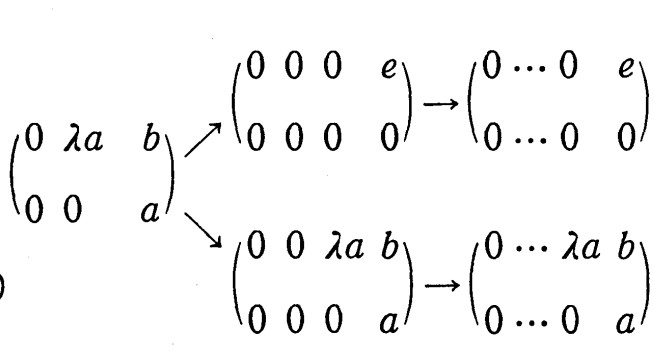 & 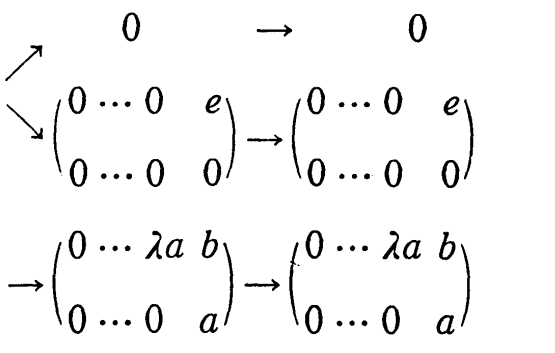 \\
\hline $\begin{array}{l}(19 \mathrm{~b}) \\
(N>2)\end{array}$ & $\left(\begin{array}{lll}0 & \lambda d_{j j}^{j} & e_{j_{1} j_{2}} \\
0 & 0 & \Omega\left(T^{2}\right)\end{array}\right) \rightarrow\left(\begin{array}{llll}0 & 0 & 0 & e_{j_{1} j_{2} j_{3}} \\
0 & 0 & 0 & 0\end{array}\right) \rightarrow\left(\begin{array}{l}0 \\
0\end{array}\right.$ & 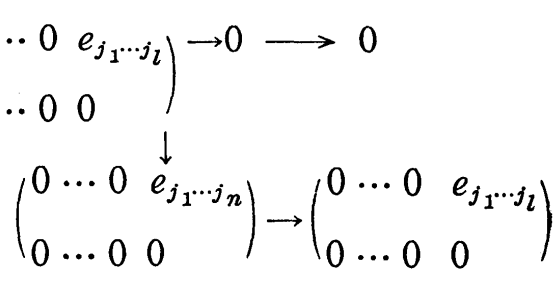 \\
\hline
\end{tabular}

\section{2. $k^{2}$}

We now begin the actual calculation of all possible kernel sequences. By lemma 11 in Chapter I, whenever $k^{1}, \cdots, k^{n}$ satisfy all requirements that can be imposed on them, we can extend to $\Lambda^{n+1} k^{n}, \cdots$, and get a valid kernel sequence. Most sequences are determined by $k^{2}$ and $k^{3}$.

In this section we compute $k^{2}$. The only requirements to be met are $k^{2} \cong \Lambda^{2} k^{1}$ and $\left[k^{1}, k^{2}\right] \subseteq k^{2}$, so it suffices if $k^{2}$ is an invariant subspace of

$$
\left(\begin{array}{ccc}
e & e_{i} & e_{i j} \\
0 & 0 & e_{j k}^{i}
\end{array}\right)
$$

It is convenient to separate $\left(\begin{array}{cc}a & a_{i} \\ 0 & b^{i}{ }_{j}\end{array}\right)$ into $\left(\begin{array}{ll}a & a_{i} \\ 0 & 0\end{array}\right)$ and $\left(\begin{array}{ll}0 & 0 \\ 0 & b^{i}{ }_{j}\end{array}\right)$; hence LEMMA 20. $k^{2}$ is admissible if and only if it is invariant under 
(1)

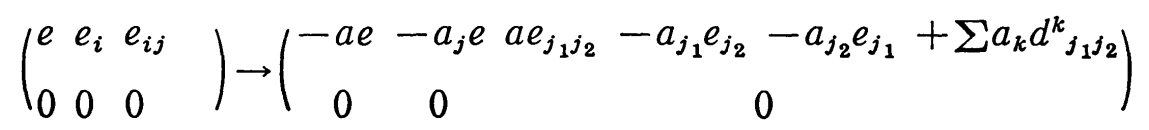

and

$$
\left(\begin{array}{ccc}
e & e_{i} & e_{i j} \\
0 & 0 & d^{i}{ }_{j_{1} j_{2}}
\end{array}\right) \rightarrow\left(\begin{array}{ccc}
0 & -\sum e_{k} b^{k}{ }_{j} & -\sum e_{j_{1}} b^{k}{ }_{j_{2}}-\sum e_{j_{2} k} b^{k}{ }_{j_{1}} \\
0 & 0 & {[d \rho(b) d]_{j_{1} j_{2}}^{i}}
\end{array}\right) .
$$

LEMMA 21. An invariant subspace of $\left(\begin{array}{lll}e & e_{i} & e_{i j} \\ 0 & 0 & d^{i}{ }_{j k}\end{array}\right)$ either has $e \equiv 0$, or has $e$ arbitrary. It either has $e_{i j} \equiv 0$, or $e_{i j}$ all symmetric tensors and arbitrary (that $i s, e_{i j}$ does not depend on $e, e_{i}$, or $d^{i}{ }_{j k}$ ).

PROOF. Under the representation (1) in lemma 20 with $a=1, a_{i}=0$,

$$
\left(\begin{array}{ccc}
e & e_{i} & e_{i j} \\
0 & 0 & d^{i}{ }_{j k}
\end{array}\right) \rightarrow\left(\begin{array}{ccc}
-e & 0 & e_{i j} \\
0 & 0 & 0
\end{array}\right) .
$$

Under the representation (2) in lemma 20, with $b^{i}{ }_{j}=\delta^{i}{ }_{j}$,

$$
\left(\begin{array}{ccc}
-e & 0 & e_{i j} \\
0 & 0 & 0
\end{array}\right) \rightarrow\left(\begin{array}{ccc}
0 & 0 & -2 e_{i j} \\
0 & 0 & 0
\end{array}\right) .
$$

Hence if $\left(\begin{array}{lll}e & e_{i} & e_{i j} \\ 0 & 0 & d^{i}{ }_{j k}\end{array}\right)$ is in an invariant subspace, so are $\left(\begin{array}{lll}e & 0 & 0 \\ 0 & 0 & 0\end{array}\right)$ and $\left(\begin{array}{lll}0 & 0 & e_{i j} \\ 0 & 0 & 0\end{array}\right)$.

Under (2) of lemma 20,

$$
\left(\begin{array}{ccc}
0 & 0 & e_{i j} \\
0 & 0 & 0
\end{array}\right) \longrightarrow\left(\begin{array}{ccc}
0 & 0 & -\sum e_{i k} b_{j}^{k}-\sum e_{k j} b_{i}^{k} \\
0 & 0 & 0
\end{array}\right),
$$

this is the Lie algebra representation corresponding to the symmetric representation of $G L(N-1, R)$, which is well-known to be irreducible. The lemma follows.

LEMMA 22. If we map an invariant subspace $k^{2}$ to $T^{2}(N-1, R)$ by

$$
\left(\begin{array}{ccc}
e & e_{i} & e_{i j} \\
0 & 0 & d^{i}{ }_{j k}
\end{array}\right) \longrightarrow d^{i}{ }_{j k}
$$

the resulting subspace of $T^{2}(N-1, R)$ is either $0,(1-\Omega)\left(T^{2}\right), \Omega\left(T^{2}\right)$, or $T^{2}$.

PROOF. This is obvious by (2) of lemma 20, and theorem 10.

LEMMA 23. Either the $e_{i}$ are arbitrary, or for any basis ${ }^{(1)} \eta^{i}{ }_{j k}, \cdots,{ }^{(r)} \eta^{i}{ }_{j k}$ of the subspace of $T^{2}(N-1, R)$ described in Lemma 22, there are vectors ${ }^{(1)} e_{i}$, $\ldots,{ }^{(r)} e_{i}$ such that whenever $d^{i}{ }_{j k}=\sum \lambda_{s}{ }^{\left({ }^{8}\right)} \eta^{i}{ }_{j k}, e_{i}=\sum \lambda_{s}{ }^{(s)} e_{i}$.

PROOF. Given a basis ${ }^{(1)} \eta^{i}{ }_{j k}, \cdots,{ }^{(r)} \eta^{i}{ }_{j k}$ of the $d^{i}{ }_{j k}$ space (which is $0, \Omega\left(T^{2}\right.$ ), $(1-\Omega)\left(T^{2}\right)$, or $\left.\left(T^{2}\right)\right)$, there exist elements of the form 


$$
\left(\begin{array}{ccc}
0 & { }^{(j)} e_{i} & 0 \\
0 & 0 & { }^{(j)} \eta^{i}{ }_{k s}
\end{array}\right)
$$

in $k^{2}$ because of lemma 21 , so if $d^{i}{ }_{j k}=\sum \lambda_{s}{ }^{(s)} \eta^{i}{ }_{j k}$, we have

in $k^{2}$.

$$
\left(\begin{array}{ccc}
0 & \sum \lambda_{s}^{(s)} e_{i} & 0 \\
0 & 0 & d^{i}{ }_{j k}
\end{array}\right)
$$

Suppose there is an element $\left(\begin{array}{ccc}e & e_{i} & e_{i j} \\ 0 & 0 & d^{i}{ }_{j k}\end{array}\right) \in k^{2}$ with $e_{i}$ not of this form. Then by lemma 21, $\left(\begin{array}{ccc}0 & e_{i} & 0 \\ 0 & 0 & d^{i}{ }_{j k}\end{array}\right) \in k^{2}$, and subtracting from the element above, $\left(\begin{array}{ccc}0 & \tilde{e}_{i} & 0 \\ 0 & 0 & 0\end{array}\right) \in k^{2}, \tilde{e}_{i} \neq 0$. By (2) of lemma 20, $\left(\begin{array}{ccc}0 & -\sum \tilde{e}_{k} b^{k}{ }_{j} & 0 \\ 0 & 0 & 0\end{array}\right) \in k^{2}$. Since this representation is irreducible, $e_{i}$ is arbitrary.

LEMMA 24. If $e \neq 0, e_{i}$ and $e_{i j}$ must be arbitrary.

Proof. By lemma 21, $\left(\begin{array}{lll}1 & 0 & 0 \\ 0 & 0 & 0\end{array}\right) \in k^{2}$. Then (1) of lemma 20, with $a=0$, $a_{i}=1$, gives $\left(\begin{array}{ccc}0 & e_{j} & 0 \\ 0 & 0 & 0\end{array}\right) \in k^{2} . \quad e_{j}=-\delta^{i}{ }_{j}$, and so $e_{j}$ is arbitrary. (1) of lemma 20 applied to the above element yields

$$
\left(\begin{array}{lll}
0 & 0 & e_{j k} \\
0 & 0 & 0
\end{array}\right)
$$

with $e_{j k}=2 \delta^{i}{ }_{j} \delta^{i}$ and so $e_{j k}$ is arbitrary.

The calculation of $k^{2}$ will now be completed by examining the various possibilities case by case.

CASE I. $\quad d_{j k}^{i} \equiv 0$. Then the possibilities are

$$
\begin{gathered}
\left(\begin{array}{lll}
e & 0 & 0 \\
0 & 0 & 0
\end{array}\right)\left(\begin{array}{ccc}
0 & e_{i} & 0 \\
0 & 0 & 0
\end{array}\right)\left(\begin{array}{lll}
0 & 0 & e_{i j} \\
0 & 0 & 0
\end{array}\right)\left(\begin{array}{lll}
e & e_{i} & 0 \\
0 & 0 & 0
\end{array}\right) \\
\left(\begin{array}{ccc}
e & 0 & e_{i j} \\
0 & 0 & 0
\end{array}\right)\left(\begin{array}{ccc}
0 & e_{i} & e_{i j} \\
0 & 0 & 0
\end{array}\right)\left(\begin{array}{ccc}
e & e_{i} & e_{i j} \\
0 & 0 & 0
\end{array}\right)\left(\begin{array}{lll}
0 & 0 & 0 \\
0 & 0 & 0
\end{array}\right) .
\end{gathered}
$$

Examine lemma 20. The first two are not invariant by (1) of this lemma. The third is invariant. The next two fail the test of lemma 24 . The last three are invariant. We have just verified $k^{2}$ for (1), (2), (7), and (12) in the list of kernels. From now on we tacitly assume $d^{i}{ }_{j k} \neq 0$.

CASE II. $e \neq 0$.

By lemma 24, we can only have

$$
\left(\begin{array}{ccc}
e & e_{j} & e_{j k} \\
0 & 0 & T^{2}
\end{array}\right)\left(\begin{array}{ccr}
e & e_{j} & e_{j k} \\
0 & 0 & \Omega\left(T^{2}\right)
\end{array}\right) \text { and }\left(\begin{array}{ccc}
e & e_{j} & e_{j k} \\
0 & 0 & (1-\Omega)\left(T^{2}\right)
\end{array}\right)
$$


Each of these is clearly invariant. They account for (13), (14), (15), and (16) on the list.

CASE III. $e \equiv 0, e_{i j}$ arbitrary.

One possibility is that $e_{i}$ is also arbitrary. This leads to

$$
\left(\begin{array}{ccr}
0 & e_{i} & e_{i j} \\
0 & 0 & \Omega\left(T^{2}\right)
\end{array}\right)\left(\begin{array}{llc}
0 & e_{i} & e_{i j} \\
0 & 0 & (1-\Omega)\left(T^{2}\right)
\end{array}\right)\left(\begin{array}{ccc}
0 & e_{i} & e_{i j} \\
0 & 0 & T^{2}
\end{array}\right),
$$

which are invariant and account for (8), (9), (10), (11) of the list.

Otherwise, we have

$$
\left(\begin{array}{ccc}
0 & \sum \lambda_{s}^{(s)} e_{j} & e_{j k} \\
0 & 0 & \sum \lambda_{s}{ }^{(s)} \eta^{i}{ }_{j k}
\end{array}\right)
$$

in the notation of lemma 23.

If all $\lambda_{s} \equiv 0$, we have

$$
\left(\begin{array}{ccc}
0 & 0 & e_{j k} \\
0 & 0 & \Omega\left(T^{2}\right)
\end{array}\right)\left(\begin{array}{ccc}
0 & 0 & e_{j k} \\
0 & 0 & (1-\Omega)\left(T^{2}\right)
\end{array}\right)\left(\begin{array}{ccc}
0 & 0 & e_{j k} \\
0 & 0 & T^{2}
\end{array}\right),
$$

all invariant. These yield (3), (4), (5), and (6) of the list. Otherwise $\lambda_{s}$ is not $\equiv 0$.

For every $d^{i}{ }_{j k}$ in the appropriate space, $\Sigma \lambda_{s}{ }^{(8)} e_{j}$ is an associated vector; thus we have a linear map from a subspace of $T^{2}$ to $R^{N-1}$; since the most general such map is

$$
e_{i}=\sum_{j k s}^{(i)} \lambda^{j}{ }_{k s} d_{k s}^{j}
$$

we have a new expression for $\sum \lambda_{s}{ }^{(s)} e_{i}$.

Thus

$$
k^{2}=\left(\begin{array}{ccc}
0 & \Sigma^{(i)} \lambda_{k l}^{j} d_{k l}^{j} & e_{j k} \\
0 & 0 & d^{i}{ }_{j k}
\end{array}\right) .
$$

The only restriction posed by lemma 20 is given by (2) and can be written:

$$
-\sum_{r j k s}{ }^{r} \lambda_{k s}^{j} d_{k s}^{j} b_{t}^{r}=\sum_{j k s}^{t} \Lambda^{j}{ }_{k s}[d \rho(b) d]^{j}{ }_{k s}
$$

CASE IIIa. $N=2$.

Then $-\lambda d b=\lambda(b d-2 b d)$, and

$$
k^{2}=\left(\begin{array}{ccc}
0 & \lambda d & e \\
0 & 0 & d
\end{array}\right)
$$

is invariant. This gives (18) and (18b) on the list.

CASE IIIb. $N>2, d^{i}{ }_{j k} \in \Omega\left(T^{2}\right)$. 
Then

$$
d^{i}{ }_{j k}=\frac{1}{2}\left[\delta^{i}{ }_{j} d_{k k}^{k}+\delta^{i}{ }_{k} d^{j}{ }_{j j}\right] .
$$

LEMMA 25. If $d$ has the above form,

$$
[\rho(b) d]_{n p}^{m}=-\frac{1}{2}\left[\delta_{n}^{m}\left(\sum d_{k k}^{k} b_{p}^{k}\right)+\delta_{p}^{m}\left(\sum d_{k k}^{k} b_{n}^{k}\right)\right]
$$

Proof. Calculate.

Using this result, the boxed-in statement above becomes

$$
\sum_{j k n}^{r} \lambda_{j n}^{j} d_{k k}^{k} b_{n}^{k}=\sum_{j k n}^{n} \lambda_{j k}^{j} d_{k k}^{k} b_{r}^{n}
$$

But

$$
\sum^{i} \lambda^{j}{ }_{k s} d^{j}{ }_{k s}=\sum_{j k s}{ }^{i} \lambda^{j}{ }_{k s} \frac{1}{2}\left(\delta^{j}{ }_{k} d^{s}{ }_{s s}+\delta^{j}{ }_{s} d_{k k}^{k}\right)=\sum_{k s}{ }^{i} \lambda^{k}{ }_{k s} d^{s}{ }_{s s} .
$$

If we define ${ }^{i} \lambda_{s}=\sum_{k}{ }^{i} \lambda^{k}{ }_{k s}$, then

$$
k^{2}=\left(\begin{array}{ccc}
0 & \Sigma^{i} \lambda_{s} d_{s s}^{s} & e_{i j} \\
0 & 0 & d^{i}{ }_{j k}
\end{array}\right)
$$

where $d^{i}{ }_{j k} \in \Omega\left(T^{2}\right)$ and

$$
\sum_{k n}^{r} \lambda_{n} d_{k k}^{k} b_{n}^{k}=\sum_{k n}{ }^{n} \lambda_{k} d_{k k}^{k} b_{r}^{n}
$$

On the other hand, $d^{k}{ }_{k k}$ can be an arbitrary vector when $d_{j k}^{i} \in \Omega\left(T^{2}\right)$, so letting $d^{k}{ }_{k k}=0$ if $k \neq s$ and 1 if $k=s$, we have

$$
\sum_{n}^{r} \lambda_{n} b^{s}{ }_{n}=\sum_{n}^{n} \lambda_{s} b^{n}{ }_{r}
$$

Letting $b^{i}{ }_{j}=\delta^{s}{ }_{i} \delta^{r}{ }_{j}$, we have ${ }^{r} \lambda_{r}={ }^{s} \lambda_{s}$. Letting $b^{i}{ }_{j}=\delta^{s}{ }_{i} \delta^{s}{ }_{j}$ we have ${ }^{r} \lambda_{s}={ }^{s} \lambda_{s} \delta^{s}{ }_{r}$, so if $r \neq s,{ }^{r} \lambda_{s}=0$. Hence $\Sigma{ }^{i} \lambda_{s} d^{s}{ }_{s s}={ }^{i} \lambda_{i} d^{i}{ }_{i i}=\lambda d^{i}{ }_{i i}$, so

$$
k^{2}=\left(\begin{array}{ccc}
0 & \lambda d^{i}{ }_{i i} & e_{j k} \\
0 & 0 & d_{j k}^{i}
\end{array}\right)
$$

where $d^{i}{ }_{j k} \in \Omega\left(T^{2}\right)$. But this is invariant, for the last boxed-in formula is easily verified. We obtain (19) and (19b) on the list.

CASE IIIC. $N>2, d^{i}{ }_{j k} \in(1-\Omega)\left(T^{2}\right)$.

LEMMA 26. Let $\alpha \neq \beta, \alpha \neq \nu, \beta \neq \nu$. Then ${ }^{r} \lambda^{\alpha}{ }_{\beta \nu}=0$.

ProOF. Say $d^{\alpha}{ }_{\beta \nu}=d^{\alpha}{ }_{\nu \beta}=1, d^{i}{ }_{j k}=0$ otherwise. Then $\Sigma d^{i}{ }_{i k}=0$, so $d^{i}{ }_{j k}$ $\in(1-\Omega)\left(T^{2}\right)$. The required formula in the box just before case IIIa can be written, for this $d$,

$$
-2 \sum_{s}^{s} \lambda^{\alpha}{ }_{\beta \nu} b_{r}^{s}=2 \sum_{m}^{r} \lambda^{m}{ }_{\beta \nu} b_{\alpha}^{m}-2 \sum_{m}^{r} \lambda_{m \nu}^{\alpha} b_{\beta m}-2 \sum_{m}^{r} \lambda_{m \beta}^{\alpha} b_{\nu m} .
$$


Letting $b_{\alpha \alpha}=1, b_{i j}=0$ otherwise, we obtain

$$
-2^{\alpha} \lambda^{\alpha}{ }_{\beta \nu} b_{\alpha r}=2^{r} \lambda^{\alpha}{ }_{\beta \nu} \text {. }
$$

If $r \neq \alpha,{ }^{r} \lambda^{\alpha}{ }_{\beta \nu}=0$. If, however, $r=\alpha$, it is still true that ${ }^{r} \lambda^{\alpha}{ }_{\beta \nu}=0$.

LEMMA 27. Let $\alpha \neq \beta, \alpha \neq \nu$. Then ${ }^{r} \lambda^{\alpha}{ }_{\beta \nu}=0$.

Proof. If $\beta \neq \nu$, this is Lemma 26. Otherwise, let $d^{\alpha}{ }_{\beta \beta}=1, d^{i}{ }_{j k}=0$ the rest of the time. This is in $(1-\Omega)\left(T^{2}\right)$ and a calculation shows that the required formula now takes the form

$$
-\sum_{\delta}^{s} \lambda^{\alpha}{ }_{\beta \beta} b_{r}^{s}=\sum_{m}^{r} \lambda^{m}{ }_{\beta \beta} b_{\alpha}^{m}-2 \sum_{n}^{r} \lambda^{\alpha}{ }_{n \beta} b_{\beta n} .
$$

Letting $b_{\beta \beta}=1, b^{i}{ }_{j}=0$ otherwise, we have

$$
-{ }^{\beta} \lambda^{\alpha}{ }_{\beta \beta} \delta^{\beta}{ }_{r}=-2^{r} \lambda^{\alpha}{ }_{\beta \beta} \text {. }
$$

If $r \neq \beta,{ }^{r} \lambda^{\alpha}{ }_{\beta \beta}=0$. If $r=\beta$, it is still true that ${ }^{r} \lambda^{\alpha}{ }_{\beta \beta}=0$.

LEMMA 28. If $\alpha \neq \beta,{ }^{r} \lambda^{\alpha}{ }_{\alpha \beta}=\frac{1}{2}{ }^{r} \lambda^{\beta}{ }_{\beta \beta}$.

PROOF. Let $d^{1}{ }_{11}=1, d^{r}{ }_{r 1}=d^{r}{ }_{1 r}=-1$ (since $N>2$, we can pick $r \neq 1$ ). Then this is in $(1-\Omega)\left(T^{2}\right)$, and the required formula becomes

$$
\begin{aligned}
-\sum_{s}\left({ }^{s} \lambda^{1}{ }_{11}-2^{s} \lambda^{r}{ }_{1 r}\right) b_{s t}= & \sum_{m}{ }^{t} \lambda^{m}{ }_{11} b^{m}{ }_{1} \\
& -2 \sum_{m}{ }^{t} \lambda^{m}{ }_{1 r} b^{m}{ }_{r}-2 \sum_{n}{ }^{t} \lambda^{1}{ }_{n 1} b^{1}{ }_{n} \\
& +2 \sum_{n}{ }^{t} \lambda^{r}{ }_{n r} b^{1}{ }_{n}+2 \sum_{n}{ }^{t} \lambda^{r}{ }_{n 1} b_{r n} .
\end{aligned}
$$

Now we apply $s \lambda^{\alpha}{ }_{\beta \nu}=0$ if $\alpha \neq \beta, \alpha \neq \nu$ to conclude that the above is

$$
\begin{aligned}
-\sum_{s}\left({ }^{s} \lambda^{1}{ }_{11}-2^{s} \lambda^{r}{ }_{1 r}\right) b^{s}{ }_{t}= & { }^{t} \lambda^{1}{ }_{11}-2^{t} \lambda^{1}{ }_{1 r} b^{1}{ }_{r} \\
& -2 \sum_{n}{ }^{t} \lambda^{1}{ }_{n 1} b^{1}{ }_{n}+2 \sum_{n}{ }^{t} \lambda^{r}{ }_{n r} b^{1}{ }_{n} .
\end{aligned}
$$

Letting $b^{1}{ }_{1}=1, b^{i}{ }_{j}=0$ otherwise, we get

$$
-\left({ }^{1} \lambda^{1}{ }_{11}-2^{1} \lambda^{r}{ }_{1 r}\right) \delta^{1}{ }_{s}={ }^{s} \lambda^{1}{ }_{11}-2^{s} \lambda^{1}{ }_{11}+2^{s} \lambda^{r}{ }_{1 r}
$$

Picking $s \neq 1,{ }^{s} \lambda^{1}{ }_{11}=2^{s} \lambda^{r}{ }_{r 1}$, so ${ }^{s} \lambda^{r}{ }_{r 1}=\frac{1}{2} s \lambda^{1}{ }_{11}$ (if $s \neq 1$ ). Since 1 and $r$ are not sacred,

$$
{ }^{\nu} \lambda^{\alpha}{ }_{\alpha \beta}=\frac{1}{2} \nu \lambda_{\beta \beta}^{\beta} \quad \text { if } \quad \nu \neq \beta .
$$

Now put $b^{1}{ }_{r}=1, b^{i}{ }_{j}=0$ otherwise, and let $s=r$. Then

$$
{ }^{1} \lambda^{1}{ }_{11}=4^{r} \lambda^{1}{ }_{1 r}+2^{1} \lambda^{r}{ }_{r 1}-2^{r} \lambda^{r}{ }_{r r} \text {. }
$$

Exchanging 1 and $r$, and putting the result for ${ }^{r} \lambda^{r}{ }_{r r}$ in the above formula, we get ${ }^{1} \lambda^{1}{ }_{11}=2{ }^{1} \lambda^{r}{ }_{r 1}$, so ${ }^{1} \lambda^{r}{ }_{r 1}=\frac{1}{2}{ }^{1} \lambda^{1}{ }_{11}$. Hence the above result holds in general 
and the lemma is proved.

LEMMA 29.

$$
{ }^{i} \lambda^{j}{ }_{k s}=\frac{1}{2}\left(\delta^{j}{ }_{k}{ }^{i} \lambda_{s s}^{s}+\delta^{j}{ }_{s}{ }^{i}{ }^{k}{ }_{k k}\right) .
$$

ProOF. A consequence of the above lemmas.

LEMMA 30. Case IIIc does not occur.

PROOF.

$$
\begin{aligned}
\sum^{i} \lambda^{j}{ }_{k s} d_{k s}^{j} & =\sum_{j k s} \frac{1}{2}\left(\delta^{j}{ }_{k}{ }^{i} \lambda^{s}{ }_{s s}+\delta^{j}{ }_{s}{ }^{i} \lambda^{k}{ }_{k k}\right) d^{j}{ }_{k s} \\
& =\sum_{k s}{ }^{i} \lambda^{s}{ }_{s s} d_{k s}^{k} .
\end{aligned}
$$

But $d^{i}{ }_{j k} \in(1-\Omega)\left(T^{2}\right)$, so $\Sigma d_{k s}^{k}=0$, so $e_{i} \equiv 0$. However this case has already been considered.

CASE IIId. $N>2, d^{i}{ }_{j k} \in T^{2}$.

In this case by lemma $17, d=d^{(1) i}{ }_{j k}+d_{j k}^{(2) i}, d^{(1)} \in(1-\Omega)\left(T^{2}\right), d^{(2)} \in \Omega\left(T^{2}\right)$.

But we can apply all the restrictions on ${ }^{i} \lambda^{j}{ }_{k s}$ proved in case IIIc. Hence

$$
e_{i}=\sum_{k s}^{i} \lambda_{s s}^{s} d_{k s}^{k}
$$

This vanishes for $d^{(1)}$, so

$$
\begin{aligned}
e_{i} & =\frac{1}{2} \sum_{k s} \lambda^{i}{ }_{s s}\left(\delta_{k}^{k} d^{(2) s}{ }_{s s}+\delta^{k}{ }_{s} d^{(2) k}{ }_{k k}\right) \\
& =\frac{N}{2}{ }^{i} \lambda_{s s}^{s} d^{(2) s}{ }_{s s} .
\end{aligned}
$$

But ${ }^{i} \lambda^{j}{ }_{k s}$ must also satisfy the conditions required when $d^{i}{ }_{j k} \in \Omega\left(T^{2}\right)$. Thus if ${ }^{i} \lambda_{j}=\sum_{k}{ }^{i} \lambda^{k}{ }_{k j},{ }^{i} \lambda_{j}=\lambda \delta^{i}{ }_{j}$ for a fixed $\lambda$. But this is just

$$
\frac{1}{2} \sum_{k}\left(\delta^{k}{ }_{k}{ }^{i} \lambda^{j}{ }_{j j}+\delta^{k}{ }_{j}{ }^{i} \lambda^{k}{ }_{k k}\right)=\frac{N}{2}{ }^{i} \lambda^{j}{ }_{j j}=\delta^{i}{ }_{j} \lambda .
$$

Hence $e_{i}=\lambda d^{(2) i}{ }_{i i}$. A simple calculation shows that this gives an invariant subspace, and yields (17) on the list.

CASE IV. $e \equiv 0, e_{j k} \equiv 0$.

Suppose $e_{i}$ is arbitrary. Then $\left(\begin{array}{ccc}0 & \delta^{i}{ }_{j} & 0 \\ 0 & 0 & 0\end{array}\right) \in k^{2}$, so by (1) of lemma 20 , $\left(\begin{array}{ccc}0 & 0 & -2 \delta^{i}{ }_{j} \delta^{i}{ }_{k} \\ 0 & 0 & 0\end{array}\right) \in k^{2}$, for each fixed $i$, a contradiction.

Hence $k^{2}$ must be $\left(\begin{array}{ccc}0 & \sum \lambda_{k}{ }^{k} e_{i} & 0 \\ 0 & 0 & \sum \lambda_{k}{ }^{k} \eta^{i}{ }_{j_{1} j_{2}}\end{array}\right)$ in the notation of lemma 23.

We must determine when this subspace is invariant. Consider first the condition that the representation preserve the relation between $e_{i}$ and $d^{i}{ }_{j k}$; but in case III we saw that this can occur only if we have 


$$
\left(\begin{array}{ccc}
0 & \lambda d^{(2) j}{ }_{j j} & 0 \\
0 & 0 & T^{2}
\end{array}\right) \text { or }\left(\begin{array}{ccr}
0 & \lambda d^{j}{ }_{j j} & 0 \\
0 & 0 & \Omega\left(T^{2}\right)
\end{array}\right) \text {. }
$$

In order that these be invariant it suffices, by lemma 20 , if

$$
-a_{j_{1}} e_{j_{2}}-a_{j_{2}} e_{j_{1}}+\sum a_{k} d_{j_{1} j_{2}}^{k}=0 .
$$

Thus if $d_{j_{1} j_{2}}^{i} \in T^{2}$, we require

$$
-\lambda a_{j_{1}} d^{(2) j_{2} j_{2} j_{2}}-\lambda a_{j_{2}} d^{(2) j_{j_{1} j_{1}}}+\sum a_{k} d_{j_{1} j_{2}}^{k}=0 .
$$

Say first $N=2$. Then this condition says

$$
-\lambda a d-\lambda a d+a d=0
$$

or $\lambda=\frac{1}{2}$. This is (18a) on the list.

If $N>2$, let $d^{2}{ }_{22}=1, d^{3}{ }_{32}=d^{3}{ }_{23}=-1$, all other $d^{i}{ }_{j k}=0$. Then $d_{j_{1} j_{2}}$ $\in(1-\Omega)\left(T^{2}\right)$, so $d^{(2){ }_{j j}}=0$, and if $a_{k}=\delta^{2}{ }_{k}$, we get a non-zero term above. Hence $T^{2}$ does not occur.

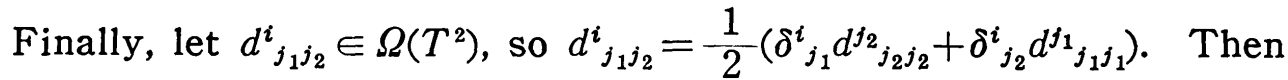

$$
0=-\lambda a_{j_{1}} d_{j_{2} j_{2}}-\lambda a_{j_{2}} d^{j_{1} j_{j_{1} j_{1}}}+\frac{1}{2} a_{j_{1}} d_{j_{2} j_{2}}+\frac{1}{2} a_{j_{2}} d^{j_{1} j_{j_{1} j_{1}}}
$$

or $\lambda=\frac{1}{2}$; this is $(18 \mathrm{~b})$ on the list.

\section{3. $k^{n}$}

The first step in the computation of $k^{n}$ is to find $\Lambda^{3} k^{2}, \cdots$ for the $k^{2}$ 's produced in the last section. The results can be seen in the list of kernels; if $k^{2}$ gives rise to several sequences, it is the last which takes the form $\Lambda^{3} k^{2}, \cdots$. By lemma 11 , each such sequence is admissible.

LEMMA 31. Let $\left(\begin{array}{lllll}0 & \cdots & 0 & e_{j_{1} \cdots j_{n}} \\ 0 & \cdots & 0 & 0\end{array}\right) \in k^{n}, e_{j_{1} \cdots j_{n}} \neq 0$. Then all possible symmetric $e_{j_{1} \cdots j_{n}}$ occur.

PROOF. Let the above element be $B^{i}{ }_{j_{1} \cdots j_{n}}$. Then

$$
\left[d \rho\left(A^{i}{ }_{j}\right) B\right]^{1}{ }_{j_{1} \cdots j_{n}}=\sum A_{{ }_{k}}^{i} B^{k}{ }_{j_{1} \cdots j_{n}}-\sum B^{1}{ }_{j_{1} \cdots k \cdots j_{n}} A_{j_{\lambda}}^{k} \text {. }
$$

Let $A=\left(\begin{array}{ll}0 & 0 \\ 0 & b^{i}{ }_{j}\end{array}\right)$; we obtain $-\sum e_{j_{1} \cdots \cdots \cdots j_{n}} b^{k}{ }_{j_{\lambda}}$. This representation on symmetric tensors is irreducible, so the result follows.

LEMMA 32. Let $A=\left(\begin{array}{cccc}0 & \cdots & 0 & e_{j_{1} \cdots j_{n}} \\ 0 & \cdots & 0 & 0\end{array}\right) \in k^{n}, B=\left(\begin{array}{lllll}0 & \cdots & 0 & e_{j_{1} \cdots j_{m}} \\ 0 & \cdots & 0 & 0\end{array}\right) \in k^{m}, m \geqq 2, n \geqq 2$. Then $[A, B]=0$.

PROOF. Look at $\Lambda^{n} \cdots \Lambda^{3} k^{2}$ for (2) on the list of kernels. Applying lemma 11 of chapter I, we conclude that $[A, B]^{1}{ }_{j_{1} \cdots j_{n}}\left(j_{i} \geqq 2\right)$ is the only non-zero 
component of $[A, B]$.

But if $n$ and $m$ are $\geqq 2$,

$$
[A, B]_{j_{1} \cdots j_{n+m-1}}^{i}=\mathcal{S} \sum A_{{ }_{k j_{1} \cdots j_{n-1}}} B_{{ }_{j_{n} \cdots j_{n+m-1}}}-\mathcal{S} \sum B_{{ }_{k j_{1} \cdots j_{m-1}}} A_{j_{m} \cdots j_{n+m-1}}^{k_{n+1}} .
$$

Since $A^{i}{ }_{j_{1} \cdots j_{n}} \neq 0$ only if $i=1, j_{i} \geqq 2$ (and similarly for $B$ ) the lemma is obvious.

We shall organize our discussion of $k^{n}$ by examining each case of the list of kernels in turn, breaking our discussion into a series of lemmas. (1) is done, and (2) is clear by the two lemmas above.

For (3), the top is an admissible sequence by lemma 11 and the fact that $\left[k^{2}, k^{2}\right] \cong k^{3}$, since

$$
\begin{aligned}
{[A, B]^{2 \cdots 2}{ }_{2 \cdots 2} } & =\mathcal{S} \sum A_{{ }_{k j_{1}}}^{2} B_{j_{2} j_{3}}^{k}-\mathcal{S} \sum B_{{ }_{k j_{1}}} A_{j_{2} j_{3}}^{k} \\
& =3\left(A^{2}{ }_{22} B^{2}{ }_{22}-B^{2}{ }_{22} A^{2}{ }_{22}\right)=0 .
\end{aligned}
$$

To complete (3), we prove that the requirement $\left[k^{2}, k^{n-1}\right] \leqq k^{n}$ forces us to adopt one of the two sequences listed in (3) of the kernel list.

Look first at the top sequence; let $A=\left(\begin{array}{ccc}0 & 0 & 0 \\ 0 & 0 & 1\end{array}\right), B=\left(\begin{array}{cccc}0 & \cdots & 0 & 1 \\ 0 & \cdots & 0 & 0\end{array}\right)$; then $[A, B]=\left(\begin{array}{cccc}0 & \cdots & 0 & * \\ 0 & \cdots & 0 & 0\end{array}\right)$ and $*=[A, B]^{1}{ }_{2 \cdots 2}=S \sum A^{1}{ }_{k 2} B^{k}{ }_{2 \cdots 2}-S \sum B^{1}{ }_{k 2 \cdots 2} A^{k}{ }_{22}=-S B^{1}{ }_{2 \cdots 2} A^{2}{ }_{22}$ $=-$ (non-zero constant).

This calculation works for the bottom sequence also, and shows that $a$ cannot be zero. Now let $A=\left(\begin{array}{lll}0 & 0 & 0 \\ 0 & 0 & 1\end{array}\right), B=\left(\begin{array}{llll}0 & \cdots & 0 & 0 \\ 0 & \cdots & 0 & 1\end{array}\right), A \in k^{2}, B \in k^{n-1}$. Say $n-1 \geqq 3$. We prove $[A, B]_{2 \cdots 2}^{2} \neq 0$, completing the calculation. Indeed

$$
\begin{aligned}
{[A, B]_{2 \cdots 2}^{2} } & =\mathcal{S} \sum A_{{ }_{k 2}}^{2} B^{k}{ }_{2 \cdots 2}-\mathcal{S} \sum B_{{ }_{k 22 \cdots 2}}^{2} A^{k}{ }_{22} \\
& =n A^{2}{ }_{22} B^{2}{ }_{2 \cdots 2}-\frac{n(n-1)}{2} A^{2}{ }_{22} B^{2}{ }_{2 \cdots 2} .
\end{aligned}
$$

But $n-\frac{n(n-1)}{2}=0 \Leftrightarrow n=0$ or $n=3$, whereas we have assumed $n \geqq 4$.

LEMMA 33. Let $A=\left(\begin{array}{llll}0 & \cdots & 0 & 0 \\ 0 & \cdots & 0 & d^{i}{ }_{j_{1} \cdots j_{n}}\end{array}\right) \in k^{n}, B=\left(\begin{array}{llll}0 & \cdots & 0 & 0 \\ 0 & \cdots & 0 & f^{i}{ }_{j_{1} \cdots j_{m}}\end{array}\right) \in k^{m} n, m \geqq 2$. Then $[A, B]=\left(\begin{array}{cccc}0 & \cdots & 0 & 0 \\ 0 & \cdots & 0 & *\end{array}\right)$ where $*$ is simply $[d, f]$ in $R^{N-1}$.

PROOF.

$$
\begin{aligned}
{[A, B]_{j_{1} \cdots j_{n+m-1}}^{i}=} & \mathcal{S} \sum A_{{ }_{k j_{1} \cdots j_{n-1}}}^{i} B_{j_{n} \cdots j_{n+m-1}}^{k} \\
& -\mathcal{S} \sum B_{{ }_{k j_{1} \cdots j_{m-1}}} A^{k_{j_{m} \cdots j_{n+m-1}}}
\end{aligned}
$$

but $A^{1}{ }_{j_{1} \cdots j_{n}}=B^{1}{ }_{j_{1} \cdots j_{m}}=0$, so the result is obvious.

Because of these lemmas and theorem 10, (4), (5), and (6) are finished if we only prove:

LEMMA 34. $d_{j_{1} j_{2}}^{i}$ and $e_{j_{1} \cdots j_{n-1}}$ can be chosen so that 


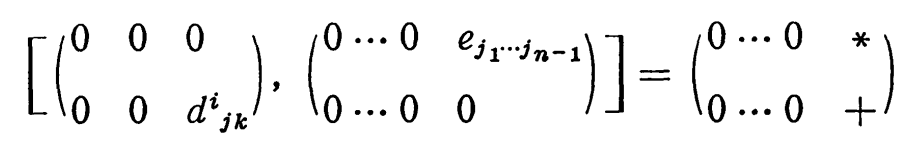

with $* \neq 0, d^{i}{ }_{j k}$ in $\Omega\left(T^{2}\right),(1-\Omega)\left(T^{2}\right)$, or $T^{2}$ (provided $N \geqq 3$ in the case $d^{i}{ }_{j k}$ $\left.\in(1-\Omega)\left(T^{2}\right)\right)$.

ProOF.

$$
\begin{aligned}
{[A, B]^{1}{ }_{j_{1} \cdots j_{n}} } & =\mathcal{S} \sum_{k} A^{1}{ }_{k j_{1}} B^{k}{ }_{j_{2} \cdots j_{n}}-\mathcal{S} \sum_{k} B^{1}{ }_{k j_{1} \cdots j_{n-2}} A^{k}{ }_{j_{n-1} j_{n}} \\
& =-\mathcal{S} \sum_{k \geqq 2} B^{1}{ }_{{ }_{k j_{1} \cdots j_{n-2}}} A^{k}{ }_{j_{n-1} j_{n}} .
\end{aligned}
$$

Let $e_{j_{1} \cdots j_{n}}=1, A^{i}{ }_{j k}=\delta^{i}{ }_{j} \eta_{k}+\delta^{i}{ }_{k} \eta_{j}, \eta_{s}=1$. Then the above expression is clearly non-zero, so we are done in the cases $\Omega\left(T^{2}\right)$ and $T^{2}$.

Let $d^{2}{ }_{33}=1, d^{i}{ }_{j k}=0$ otherwise. This is in $(1-\Omega)\left(T^{2}\right)$, and clearly gives a non-zero result.

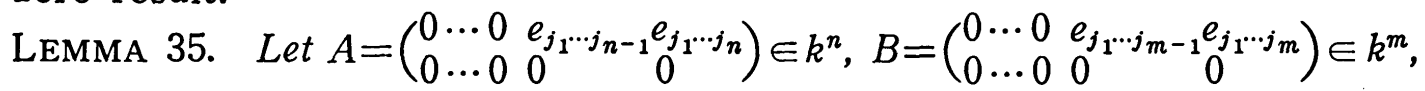
$n \geqq 2, m \geqq 2$. Then $[A, B]=\left(\begin{array}{llll}0 & \cdots & 0 & * \\ 0 & \cdots & 0 & 0\end{array}\right)$.

Proof. By (7) and lemma 11, we know $[A, B]=\left(\begin{array}{ccccc}0 & \cdots & 0 & + & * \\ 0 & \cdots & 0 & 0 & 0\end{array}\right)$. We are to prove $+=0$.

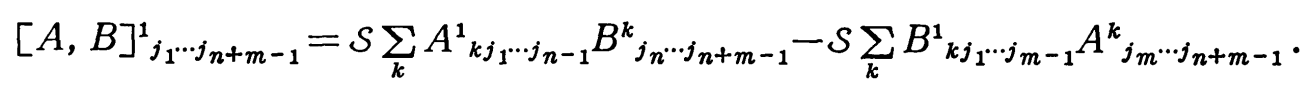

Then $k$ must be 1 . Suppose $j_{1}=1$, all other $j_{i} \geqq 2$. Since $A^{1}{ }_{11 j_{2} \cdots j_{n}}=0$, this is

$$
\mathcal{S} A^{1}{ }_{1 j_{2} \cdots j_{n}} B^{1}{ }_{1 j_{n+1} \cdots j_{n+m-1}}-\mathcal{S} B^{1}{ }_{1 j_{2} \cdots j_{m}} A^{1}{ }_{1 j_{m+1} \cdots j_{n+m-1}}
$$

(here $\mathcal{S}$ means symmetrization of $j_{2}, \cdots, j_{n+m-1}$ ). These two are clearly equal, so the result is zero.

LEMMA 36. It is possible to pick $e_{j}$ and $e_{j_{1} \cdots j_{n-1}}$ so that if

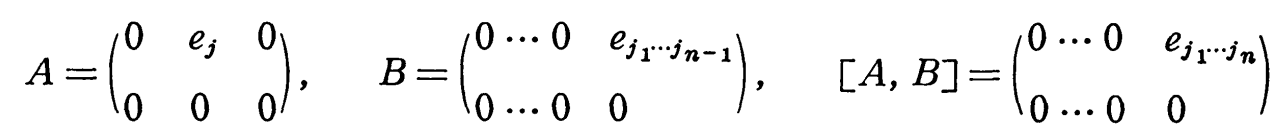

with $e_{j_{1} \cdots j_{n}} \neq=0$.

Proof. We use the formula above for $[A, B]$. Clearly if $j_{i} \geqq 2$,

$$
[A, B]^{1}{ }_{j_{1} \cdots j_{n}}=S A^{1}{ }_{1 j_{1}} B^{1}{ }_{j_{2} \cdots j_{n}} \text {. }
$$

The result is then obvious.

LEMMA 37. Let $k^{n}$ contain all $\left(\begin{array}{llll}0 & \cdots & 0 & e_{j_{1} \cdots j_{n}} \\ 0 & \cdots & 0 & 0\end{array}\right)$ and in addition some $B=\left(\begin{array}{cccc}0 & \cdots & 0 & e_{j_{1} \cdots j_{n-1}} \\ 0 & \cdots & e_{j_{1} \cdots j_{n}}\end{array}\right), e_{j_{1} \cdots j_{n-1}} \neq 0$. Then it contains all elements of this form.

PROOF. Again we look at $\left[d \rho\left(A^{i}{ }_{j}\right) B\right]^{1}{ }_{1 j_{1} \cdots j_{n-1}}, j_{i} \geqq 2$, for $A=\left(\begin{array}{ll}0 & 0 \\ 0 & b^{i}{ }_{j}\end{array}\right)$. It is 


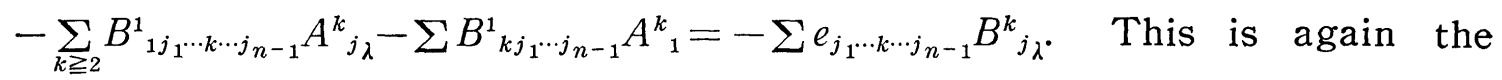
symmetric representation which is irreducible, and the result follows.

Clearly, lemmas $31,35,36,37$ complete (7).

Now turn to (8). To show that the top occurs, we must prove $\left[k^{2}, k^{2}\right]$ $\subseteq k^{3}$; but $[A, B]_{222}^{2}=\mathcal{S} \sum_{k} A^{2}{ }_{k 2} B^{k}{ }_{22}-\mathcal{S} \sum_{k} B^{2}{ }_{k 2} A^{k}{ }_{22}=3\left(A^{2}{ }_{12} B_{22}^{1}+A^{2}{ }_{22} B^{2}{ }_{22}-B^{2}{ }_{22} A^{2}{ }_{22}\right.$ $\left.-B^{2}{ }_{12} A^{1}{ }_{22}\right)=0$.

To complete (8), notice that lemma 33 and theorem 10 show that $a$ occurs in all $k^{n}, n \geqq 4$, and lemma 36 shows that $b$ always occurs. It suffices if

$$
\left[\left(\begin{array}{lll}
0 & 0 & 0 \\
0 & 0 & 1
\end{array}\right),\left(\begin{array}{lllll}
0 & \cdots & 0 & 1 & 0 \\
0 & \cdots & 0 & 0 & 0
\end{array}\right)\right]=\left(\begin{array}{lllll}
0 & \cdots & 0 & + & * \\
0 & \cdots & 0 & 0 & 0
\end{array}\right)
$$

with $+\neq 0$. This is a special case of :

LEMMA 38. It is possible to pick $d_{j k}^{i} \in \Omega\left(T^{2}\right),(1-\Omega)\left(T^{2}\right)$, or $T^{2}$ and $e_{j_{1} \cdots j_{n-1}}$ so that if

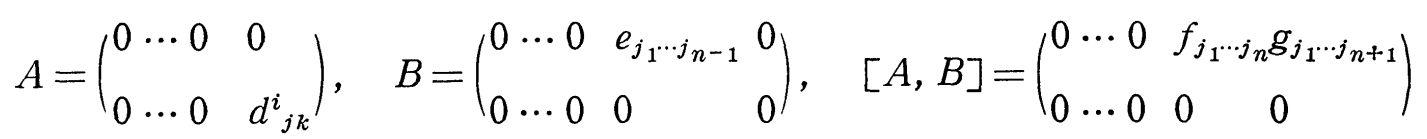

with $f_{j_{1} \cdots j_{n}} \neq 0$, provided $n \geqq 2$ and, if $d^{i}{ }_{j k} \in(1-\Omega)\left(T^{2}\right), N \geqq 3$.

PROOF. If $j_{2}, \cdots, j_{n+1} \geqq 2, j_{1}=1$,

$$
\begin{aligned}
& {[A, B]_{j_{1} j_{2} \cdots j_{n+1}}^{1}=\mathcal{S} \sum_{k} A_{k_{j} j_{1}}^{1} B_{j_{2} \cdots j_{n+1}}^{k}-\mathcal{S} \sum B^{1}{ }_{k j_{1} \cdots j_{n-1}} A_{j_{n} j_{n+1}}^{k}} \\
& =\mathcal{S} A^{1}{ }_{1 j_{1}} B^{1}{ }_{j_{2} \cdots j_{n+1}}-\mathcal{S} \sum_{k \geqq 2} B^{1}{ }_{k j_{1} \cdots j_{n-1}} A^{k}{ }_{j_{n} j_{n+1}} .
\end{aligned}
$$

Now $A^{1}{ }_{j_{1} j_{2}}=0$, so this is $-\mathcal{S} \sum_{k \geqq 2} B^{1}{ }_{k 1 j_{2} \cdots j_{n-1}} A^{k}{ }_{j_{n} j_{n+1}}$, where $\mathcal{S}$ is symmetrization of $j_{2}, \cdots, j_{n+1}$; letting $e_{j_{1} \cdots j_{n-1}}=1$ and $A^{k}{ }_{j_{1} j_{2}}=\delta^{k}{ }_{j_{1}} \eta_{j_{2}}+\delta^{k}{ }_{j_{2}} \eta_{j_{1}}$ where $\eta_{j}=1$, the result is true for $\Omega\left(T^{2}\right)$ and $T^{2}$.

Letting $d^{2}{ }_{33}=1, d^{i}{ }_{j j}=0$ otherwise, the result is also true for $(1-\Omega)\left(T^{2}\right)$.

Clearly lemmas $31,33,36,37$, and 38 complete (9), (10), and (11).

LEMma 39. If $A=\left(\begin{array}{ccc}e & e_{i} & e_{i j} \\ 0 & 0 & e^{i}{ }_{j k}\end{array}\right), B=\left(\begin{array}{ccc}f & f_{i} & f_{i j} \\ 0 & 0 & f^{i}{ }_{j k}\end{array}\right),[A, B]=\left(\begin{array}{lll}0 & * & * \\ 0 & 0 & *\end{array}\right)$.

Proof. By (16), it suffices if $[A, B]_{1 \cdots 1}^{1}=0$. But $[A, B]_{j_{1} j_{2} j_{3}}^{1}=\mathcal{S} \sum_{k} A^{1}{ }_{k j_{1}} B^{k}{ }_{j_{2} j_{3}}$ $-\mathcal{S} \sum_{k} B^{1}{ }_{k j_{1}} A^{k}{ }_{j_{2} j_{3}}=\mathcal{S} A^{1}{ }_{{ }_{1} j_{1}} B^{1}{ }_{j_{2} j_{3}}+\mathcal{S} \sum_{k \geqq 2} A^{1}{ }_{k j_{1}} B^{k}{ }_{j_{2} j_{3}}-\mathcal{S} B^{1}{ }_{j_{1}} A^{1}{ }_{j_{2} j_{3}}-\mathcal{S} \sum_{k \geqq 2} B^{1}{ }_{k j_{1}} A^{k}{ }_{j_{2} j_{3}}$. Hence $[A, B]^{1}{ }_{111}=3\left(A^{1}{ }_{11} B^{1}{ }_{11}-B^{1}{ }_{11} A^{1}{ }_{11}\right)=0$.

Clearly lemmas 33 and 39 prove that all kernel sequences in (12), (13), (14), (15), and (16) are allowed, since $\left[k^{2}, k^{2}\right] \cong k^{3}$.

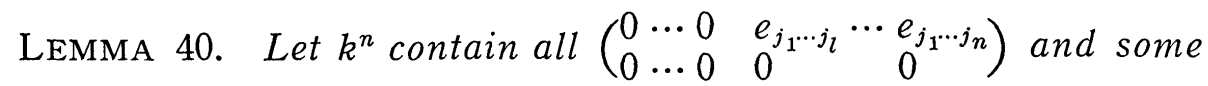

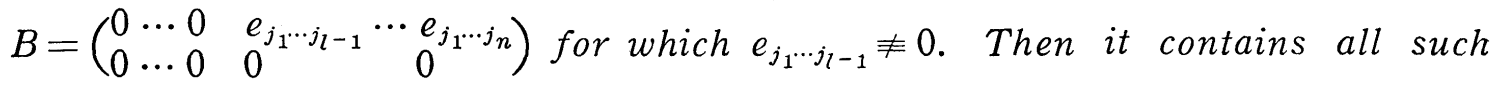


elements.

Proof. Let $A=\left(\begin{array}{ll}0 & 0 \\ 0 & b^{i}{ }_{j}\end{array}\right)$, and consider $[d \rho(A) B]^{1}{ }_{j_{1} \cdots j_{n}}=-\sum B^{1}{ }_{j_{1} \cdots \cdots \cdots j_{n}} A^{k}{ }_{j_{\lambda^{*}}}$. But $[d \rho(A) B]^{1}{ }_{11 \cdots 1 j_{1} \cdots j_{l-1}}=-\sum_{k \geqq 2} e_{j_{1} \cdots k \cdots j_{l-1}} b^{k}{ }_{j_{\lambda}}$ since $A^{k}=0$; this representation is irreducible, so we are done.

LEMMA 41. If $l \neq(n-1)-2$, it is possible to pick $e_{j_{1} \cdots j_{l}}$ so that if

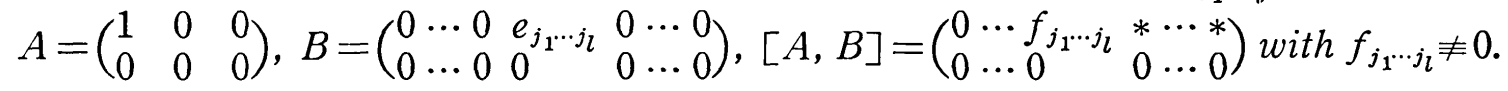

PROOF.

$$
\begin{aligned}
{[A, B]^{1}{ }_{j_{1} \cdots j_{n}} } & =\mathcal{S} \sum_{k} A^{1}{ }_{k j_{1}} B^{k}{ }_{j_{2} \cdots j_{n}}-\mathcal{S} \sum_{k} B^{1}{ }_{k j_{1} \cdots j_{n-2}} A^{k}{ }_{j_{n-1} j_{n}} \\
& =\mathcal{S} A^{1}{ }_{{ }_{1} j_{1}} B^{1}{ }_{j_{2} \cdots j_{n}}-\mathcal{S} B^{1}{ }_{1 j_{1} \cdots j_{n-2}} A^{1}{ }_{j_{n-1} j_{n}} .
\end{aligned}
$$

Now $B^{1}{ }_{j_{1} \cdots j_{n-1}} \neq 0$ only if precisely $l$ of the $j_{i}$ are $\geqq 2$. If less than $l$ of $j_{1} \cdots j_{n}$ are $\geqq 2,[A, B]_{j_{1} \cdots j_{n}}^{1}$ is zero, since for the first term $j_{i}=1$, so all $j_{i} \geqq 2$ are in $B$ and there are less than $l$ such, while in the second term $j_{n-1}$ and $j_{n}$ must be 1 , so there are less than $l$ terms $\geqq 2$ in $B$.

If exactly $l$ terms are $\geqq 2$, we have

$$
(n-l) e_{j_{1} \cdots j_{l}}-e_{j_{1} \cdots j_{l}}-\frac{(n-l)(n-l-1)}{2} .
$$

This is zero just if $(n-l)\left(1-\frac{n-l-1}{2}\right)=0$, i. e., either $l=n$ or $n-l=3$. But $l \leqq n-1$, so the only bad case is $n=3+l$.

Lemmas 33, 40, and 41 clearly complete the top sequences of (12), (14), (15), and (16), and the first and third sequences of (13).

LEMMA 42. If $l \neq n-2$, it is possible to pick $e_{i}$ and $e_{j_{1} \cdots j_{l}}$ so if

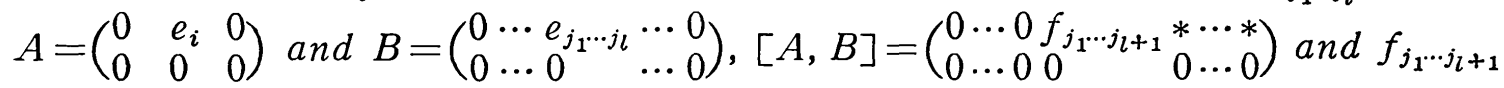
$\not \equiv 0$.

PROOF. As before $[A, B]^{1}{ }_{j_{1} \cdots j_{n}}=S A^{1}{ }_{1 j_{1}} B^{1}{ }_{j_{2} \cdots j_{n}}-\mathcal{S} B^{1}{ }_{1 j_{1} \cdots j_{n-2}} A^{1}{ }_{j_{n-1} j_{n}}$. If exactly $l+1 j$ 's are $\geqq 2$, this is

$$
S e_{j_{1}} e_{j_{2} \cdots j_{l}+1}-S e_{j_{1}} e_{j_{2} \cdots j_{l+1}}(n-(l+1)) \text {. }
$$

If $e_{i}=1, e_{j_{1} \cdots j_{l}}=1$, this can only be zero if $1-n+l+1=0$, or $n=l+2$.

When we put lemmas 41 and 42 together, we discover that if all $\left(\begin{array}{lllll}e & e_{j_{1}} & \cdots & e_{j_{1} \cdots j_{n-1}} \\ 0 & 0 & \cdots & 0\end{array}\right) \in k^{n-1}$, we get all $\left(\begin{array}{lllll}e & e_{j_{1}} & \cdots & e_{j_{1} \cdots j_{n}} \\ 0 & 0 & \cdots & 0\end{array}\right) \in k^{n}$, provided $n>3$, for lemma 41 allows us to fill in all of $k^{n}$ except $e_{j_{1} \cdots j_{l}}, l=n-3$, but lemma 42 allows us to fill in everything except $l=n-2$ and $l=0$. Only if $n=3$ do we have trouble, corresponding to the splitting observed.

It is now clear that the above lemmas, together with lemma 33, complete (12), (13), (14), (15), and (16). Let us next discuss (19) and (19b).

LEMMA 43. We can find $d^{i}{ }_{j k} \in \Omega\left(T^{2}\right)$ and $e_{j_{1} \cdots j_{n-1}}$ so that when 
$A=\left(\begin{array}{ccc}0 & \lambda d^{j}{ }_{j j} & 0 \\ 0 & 0 & d^{i}{ }_{j k}\end{array}\right)$ and $B=\left(\begin{array}{cccc}0 & \cdots & 0 & e_{j_{1} \cdots j_{n-1}} \\ 0 & \cdots & 0 & 0\end{array}\right)$ then $[A, B]=\left(\begin{array}{llll}0 & \cdots & 0 & e_{j_{1} \cdots j_{n}} \\ 0 & \cdots & 0 & 0\end{array}\right)$ with $e_{j_{1} \cdots j_{n}} \neq 0 \Leftrightarrow \lambda \neq \frac{n-1}{2}$.

Proof. We know $[A, B]$ has this form, and

$$
[A, B]^{1}{ }_{j_{1} \cdots j_{n}}=S A^{1}{ }_{1 j_{1}} B^{1}{ }_{j_{2} \cdots j_{n}}-\mathcal{S} \sum_{k \geqq 2} B^{1}{ }_{k j_{1} \cdots j_{n-2}} A^{k}{ }_{j_{n-1} j_{n}} \text {. }
$$

This is

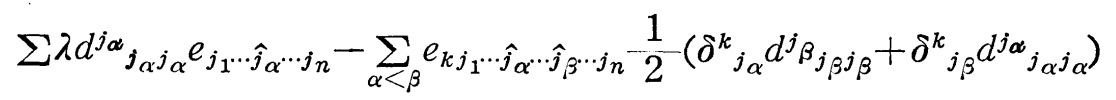

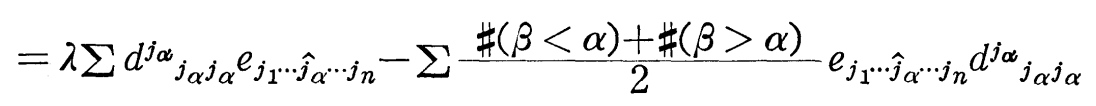

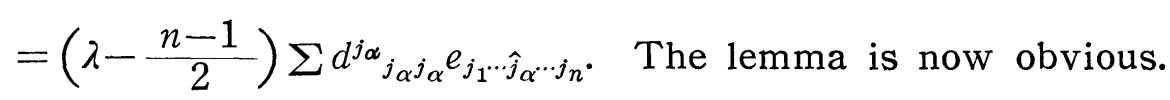

LEMMA 44. If $\lambda=1, d^{i}{ }_{j k}$ and $f^{i}{ }_{j k} \in \Omega\left(T^{2}\right)$, then

$$
\left[\left(\begin{array}{ccc}
0 & d^{j}{ }_{j j} & 0 \\
0 & 0 & d^{i}{ }_{j k}
\end{array}\right),\left(\begin{array}{ccc}
0 & f^{j}{ }_{j j} & 0 \\
0 & 0 & f^{i}{ }_{j k}
\end{array}\right)\right]=0
$$

PROOF. By (19) we need only look at $[A, B]_{j_{1} \cdots j_{n}}^{1}$ It is $\mathcal{S} \sum_{k \geqq 2} A^{1}{ }_{k j_{1}} B^{k}{ }_{j_{2} j_{3}}$ $-\mathcal{S} \sum_{k \geqq 2} B_{{ }_{k j} j_{1}} A^{k}{ }_{j_{2} j_{3}}=0$ since $A^{1}{ }_{k j}=0$ if $k \geqq 2, j \geqq 2$.

But these two lemmas together clearly imply (19) and (19b). (18a) and (19a) are complete as is. Next turn to (17). A calculation shows that $\Lambda^{n} \cdots \Lambda^{3} k^{2}=\left(\begin{array}{lllll}0 & \cdots & 0 & f\left(d^{i}{ }_{j_{1} \cdots j_{n}}\right. & \\ 0 & \cdots & 0 & 0 & e_{j_{1} \cdots j_{n}} \\ d^{i}{ }_{j_{1} \cdots j_{n}}\end{array}\right)$ with $f$ some function we need not give more explicitly.

LEMMA 45. If $A=\left(\begin{array}{cccc}e & e_{j_{1}} & \cdots & e_{j_{1} \cdots j_{n}} \\ 0 & 0 & \cdots & d^{i}{ }_{j_{1} \cdots j_{n}}\end{array}\right)$ and $B=\left(\begin{array}{cccc}f & f_{j_{1}} & \cdots & f_{j_{1} \cdots j_{n}} \\ 0 & 0 & \cdots & k_{j_{1} \cdots j_{n}}^{i}\end{array}\right)$ then $[A, B]=$ $\left(\begin{array}{llll}* & * & \cdots & * \\ 0 & 0 & \cdots & {[d, k]}\end{array}\right)$.

PROOF. A variant of lemma 33.

To finish (17), we need only prove lemma 43 without assuming $\lambda \neq \frac{n-1}{2}$ provided $d^{i}{ }_{j k}$ is allowed to vary through $T^{2}$.

But $N \geqq 3$, so pick $d^{2}{ }_{33}=1, d^{i}{ }_{j k}=0$ otherwise. Then look at

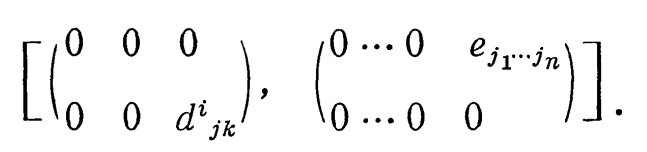

Using the formula of lemma $43,[A, B]^{1}{ }_{j_{1} \cdots j_{n}}=-\mathcal{S} \sum e_{k j_{1} \cdots j_{n-2}} d^{k}{ }_{j_{n-1} j_{n}}$. This can clearly be made non-zero and we are done.

Consider (18). The top of (18) is a sequence because $\left[k^{2}, k^{2}\right] \subseteq k^{3}$ by lemma 45 and theorem 10. Note that lemma 43 holds as $\Omega\left(T^{2}\right)=T^{2}$ for $N=2$. The bottom of (18) is a sequence by lemma 45 and theorem 10 . 
Similarly, the top of $(18 \mathrm{~b})$ is a sequence.

To conclude the discussion of (18) we need:

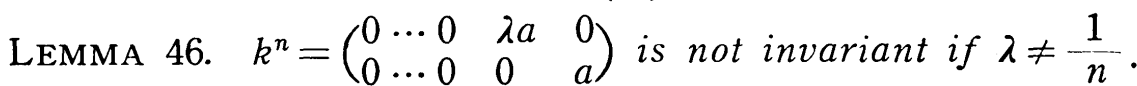

PROOF.

$$
\begin{aligned}
{[d \rho(A) B]^{1}{ }_{22 \cdots 2} } & =\Sigma A^{1}{ }_{k} B^{k}{ }_{22 \cdots 2}-n \sum B^{1}{ }_{k 22 \cdots 2} A^{k}{ }_{2} \\
& =A^{1}{ }_{2} B^{2}{ }_{22 \cdots 2}-n B^{1}{ }_{12 \cdots 2} A^{1}{ }_{2} \\
& =A^{1}{ }_{2}(a-n \lambda a) .
\end{aligned}
$$

Since $\frac{1}{n}=\frac{n-1}{2}$ implies $n=2,(18 \mathrm{~b})$ is true.

\section{University of Oregon}

\section{BIBLIOGRAPHY}

[1] S. Bochner and R.C. Gunning, Infinite linear pseudogroups of transformations, Ann. of Math., 75 (1962), 93-104.

[2] H. Boerner, Representations of groups, North-Holland Publishing Co., Amsterdam, 1963.

[3] V. Guillemin and S. Sternberg, An algebraic model of transitive differential geometry, Bull. Amer. Math. Soc., 70 (1964), 16-47.

[4] R.C. Gunning, Complex analytic pseudogroups and the quasi-uniformization of complex manifolds (unpublished).

[5] R. C. Gunning, Connections for a class of pseudogroup structures, in Proceedings of the conference on complex analysis (Minneapolis), Springer-Verlag, New York, 1965.

[6] S. Helgason, Differential geometry and symmetric spaces, Academic Press, New York, 1962.

[7] S. Kobayashi and T. Nagano, On filtered Lie algebras and geometric structures I, II, III, J. Math. and Mech., 13 (1964), 875-908; 14 (1965), 513-521, 679-706.

[8] I. M. Singer and Shlomo Sternberg, The infinite groups of Lie and Cartan, part I (the transitive groups), J. Analyse Math., 15 (1965), 4-113.

[9] H. Weyl, The classical groups, Princeton Univ. Press, Princeton, 1946. 